Preprint typeset in JHEP style - HYPER VERSION

\title{
Conformal Lifshitz Gravity from Holography
}

\author{
Tom Griffin, Petr Hořava and Charles M. Melby-Thompson \\ Berkeley Center for Theoretical Physics and Department of Physics \\ University of California, Berkeley, CA, 94720-7300 \\ and \\ Theoretical Physics Group, Lawrence Berkeley National Laboratory \\ Berkeley, CA 94720-8162, USA
}

\begin{abstract}
We show that holographic renormalization of relativistic gravity in asymptotically Lifshitz spacetimes naturally reproduces the structure of gravity with anisotropic scaling: The holographic counterterms induced near anisotropic infinity take the form of the action for gravity at a Lifshitz point, with the appropriate value of the dynamical critical exponent $z$. In the particular case of $3+1$ bulk dimensions and $z=2$ asymptotic scaling near infinity, we find a logarithmic counterterm, related to anisotropic Weyl anomaly of the dual CFT, and show that this counterterm reproduces precisely the action of conformal gravity at a $z=2$ Lifshitz point in $2+1$ dimensions, which enjoys anisotropic local Weyl invariance and satisfies the detailed balance condition. We explain how the detailed balance is a consequence of relations among holographic counterterms, and point out that a similar relation holds in the relativistic case of holography in $A d S_{5}$. Upon analytic continuation, analogous to the relativistic case studied recently by Maldacena, the action of conformal gravity at the $z=2$ Lifshitz point features in the ground-state wavefunction of a gravitational system with an interesting type of spatial anisotropy.
\end{abstract}




\section{Contents}

1. Introduction 2

2. Gravity at a Lifshitz Point 3

2.1 Anisotropic Weyl Transformations 5

$2.2 z=2$ Conformal Gravity in $2+1$ Dimensions

3. Holography in Asymptotically Lifshitz Spacetimes 7

3.1 Anisotropic Conformal Infinity

3.2 Asymptotically Lifshitz Spacetimes 9

4. Holographic Renormalization in Asymptotically Lifshitz Spacetimes 10

4.1 Hamiltonian Approach to Holographic Renormalization 10

4.2 Bulk Gravity with a Massive Vector 13

4.3 Gravity with a Massive Vector Coupled to Bulk Scalars 14

4.4 Explaining Detailed Balance 18

4.5 Analytic Continuation to the de Sitter-like Regime 18

5. Conclusions 20

A. Notation and Conventions 21

A.1 The bulk action 22

A.2 ADM decomposition in the metric formalism 22

A.3 ADM decomposition in the vielbein formalism 23

A.4 The massive vector 24

A.5 Functional derivatives and the stress tensor 24

A.6 Boundary source fields and asymptotic scaling 25

B. Holographic Renormalization Equations 27

B.1 Non-derivative counterterms 29

B.2 Two-derivative counterterms with $\psi=0$

B.3 Two-derivative counterterms involving $\psi$

B.4 Four-derivative counterterms with $\psi=0$

B.5 Four-derivative counterterms involving $\psi$

C. Anisotropic Weyl Anomaly in 2+1 Dimensions 35 


\section{Introduction}

The possibility that gravity may exhibit multicritical behavior with Lifshitz-like anisotropic scaling at short distances $[1,2]$ has attracted a lot of attention recently (see, e.g., [3-5] for some reviews). Such multicritical quantum gravity can be formulated as a field theory of the fluctuating spacetime metric, characterized by scaling which is anisotropic between time and space,

$$
t \mapsto b^{z} t, \quad \mathbf{x} \mapsto b \mathbf{x},
$$

with dynamical exponent $z$.

When $z$ equals the number of spatial dimensions $D$, several interesting things happen: First, the theory becomes power-counting renormalizable, when we allow all terms compatible with the gauge symmetries in the action. In addition, the effective spectral dimension of spacetime flows to two at short distances, in accord with the lattice results first obtained in the causal dynamical triangluations approach to quantum gravity in [6-8], and independently confirmed recently in [9]. Moreover, when $z=D$, one can further restrict the classical action by requiring its invariance under the local version of the rigid anisotropic scaling, which acts on the spacetime metric via anisotropic Weyl transformations. This leads to an anisotropic version of conformal gravity [1].

While such multicritical gravity models may not need string theory for a UV completion, it is still natural to ask whether they can be engineered from string theory. Indeed, it seems likely that any mathematically consistent theory of gravity should have a role to play in the bigger scheme of strings. Here we present one specific construction in which the action of multicritical gravity with anisotropic scaling appears naturally from string theory and AdS/CFT correspondence: The holographic renormalization of spacetimes which are asymptotically Lifshitz, or in other words, dual to nonrelativistic field theories with Lifshitz scaling.

In recent years, the AdS/CFT correspondence has been extended to spacetimes which are asymptotically non-relativistic, with the hope of providing new techniques for understanding strongly coupled condensed matter systems using dualities originating in string theory (see, e.g., [10-13] for recent reviews of this program). Such asymptotically non-relativistic spacetimes fall into two classes: Either they approach Schrödinger symmetries $[14,15]$, or they exhibit Lifshitz-type scaling [16]. In both cases, Penrose's standard definition of conformal infinity (see, e.g., [17]) gives results which are puzzling and appear inconsistent with the expectations based on gauge-gravity duality. It turns out that for spacetimes which carry an asymptotic foliation structure, a natural generalization of Penrose's notion of conformal infinity to asymptotically anisotropic spacetimes exists [18], and it reproduces features expected from holography.

This clearer picture [18] of the asymptotic structure of Lifshitz spacetimes allows us to perform holographic renormalization, study the precise structure of holographic counterterms, and compare the results to the relativistic case. This is the goal of the present paper. We focus mainly on the case of $3+1$ bulk dimensions, in particular with the $z=2$ scaling. In 
this case, we find a logarithmic counterterm, which takes the form of the action of the $z=2$ conformal multicritical gravity in $2+1$ dimensions. In relativistic AdS/CFT, logarithmic gravitational counterterms appear only when the dimension $d$ of the boundary is even. In that circumstance, they take the form of the Weyl anomaly [19] (see [20] for a review of the Weyl anomaly). For example, in the maximally supersymmetric case in $d=4$, the anomaly is given by the action of conformal supergravity [21] (see, e.g., [22] for a review of the early history of conformal supergravity). In Lifshitz spacetimes, the logarithmic counterterms - if and when they appear - should be related to the little-studied nonrelativistic Weyl anomaly (see [23] for some early results on the Weyl anomaly at $z=3$ in $d=4$, and [24,25] for a detailed discussion of axial anomalies in Lifshitz theories). In Appendix C, we briefly discuss the cohomological structure of the $z=2$ anisotropic Weyl anomaly in $2+1$ dimensions, and (modulo total derivatives) find two independent terms that can appear in the action. However, perhaps surprisingly, the action that we obtain in the logarithmic counterterm turns out to satisfy the additional condition of detailed balance, which reduces the number of independent terms to one. We show how this condition is implied by the machinery of holographic renormalization, which relates the logarithmic counterterm to another, quadratic counterterm.

The techniques of holographic renormalization in asymptotically $A d S$ spacetimes can also be usefully applied, upon appropriate Wick rotation, to asymptotically de Sitter geometries [26], leading to results about the ground-state wavefunction of the universe at superhorizon scales [27] (see also [28, 29], and the series of papers [30-32]). Since holography in asymptotically Lifshitz spacetimes parallels closely the case of $A d S$, it is natural to perform the corresponding Wick rotation, obtain a candidate ground-state wavefunction, and ask what kind of gravitational system this wavefunction describes. In the case of $z=2$ and bulk $3+1$ dimensions, we show that this wavefunction corresponds to a spatially anisotropic gravity theory with an interesting form of ultralocality.

Our discussion in the bulk of the paper mostly focuses on the case of $3+1$ bulk dimensions, in particular with $z=2$. However, after summarizing our conventions and notation in Appendix A, we present our detailed calculations also for general $D$ and $z$ in an extensive Appendix B, with the hope that the inquisitive reader may find the results useful.

\section{Gravity at a Lifshitz Point}

In this section, we briefly review some features of gravity with anisotropic scaling, concentrating on aspects relevant for the main points of this paper.

The theory can be formulated in the general number of $d=D+1$ spacetime dimensions. Since the spacetime manifold $M$ is assumed to carry a preferred foliation structure $\mathcal{F}$, consisting of codimension-one leaves $\Sigma$ of constant absolute time, it is natural to use nonrelativistic coordinates $t$ and $\mathbf{x} \equiv\left\{x^{i}, i=1, \ldots D\right\}$, adapted to the foliation. In such coordinates, the theory is then described by specifying its fields and its symmetries. The gravity field multiplet 
consists of fields

$$
N, \quad N_{i}, \quad \gamma_{i j},
$$

familiar from the ADM decomposition of the relativistic metric on spacetime: $N$ is the lapse function, $N_{i}$ the shift vector, and $\gamma_{i j}$ the spatial metric on the leaves $\Sigma$. Ocassionally, we will refer to the set of fields (2.1) as the "metric multiplet," to indicate that the ADM split is not just a convenience, but a reflection of the preferred foliation structure of spacetime. To think of the spacetime metric as one irreducible field would be misleading in the context of Lifshitz gravity.

In the simplest version of the theory, the gauge symmetries are given by those spacetime diffeomorphisms that preserve the preferred foliation $\mathcal{F}$. Such foliation-preserving diffeomorphisms $\operatorname{Diff}(M, \mathcal{F})$, generated by

$$
\delta t=f(t), \quad \delta x^{i}=\xi^{i}(\mathbf{x}, t),
$$

contain one fewer gauge symmetry per spacetime point than the symmetries of general relativity. Theories of gravity with anisotropic scaling whose symmetries are as large as those of general relativity can be constructed [33], but they will stay outside of the scope of the present paper.

The action respecting the symmetries of $\operatorname{Diff}(M, \mathcal{F})$ consists of a kinetic term,

$$
S_{K}=\frac{1}{\kappa^{2}} \int d t d^{D} \mathbf{x} \sqrt{\gamma} N\left(\hat{K}_{i j} \hat{K}^{i j}-\lambda \hat{K}^{2}\right)
$$

where

$$
\hat{K}_{i j}=\frac{1}{2 N}\left(\partial_{t} \gamma_{i j}-\nabla_{i} N_{j}-\nabla_{j} N_{i}\right)
$$

is the extrinsic curvature of $\Sigma, \hat{K} \equiv \hat{K}_{i}^{i}$, and $\lambda$ is dimensionless coupling constant; and a potential term

$$
S_{\mathcal{V}}=\frac{1}{\kappa^{2}} \int d t d^{D} \mathbf{x} \sqrt{\gamma} N \mathcal{V}
$$

with $\mathcal{V}$ a scalar function built out of the spatial Riemann tensor and its covariant spatial derivatives, but independent of the time derivatives of all fields. Among these terms, the spatial scalar curvature $R$ and the constant term dominate at long distances, while terms of scaling dimension $2 z$ take over the dynamics at shortest scales.

In higher dimensions, and for higher values of $z$, the number of available relevant and marginal terms that can appear in $\mathcal{V}$ proliferates quickly. One can further limit the independent terms by imposing an additional symmetry. For example, one can impose the detailed balance condition $[1,2]$. This condition means that $\mathcal{V}$ is constructed from an auxiliary local action $\mathcal{W}$ in $D$ Euclidean dimensions, as the sum of squares of the $\mathcal{W}$ equations of motion:

$$
\mathcal{V}=\mathcal{G}_{i j k \ell} \frac{\delta \mathcal{W}}{\delta \gamma_{i j}} \frac{\delta \mathcal{W}}{\delta \gamma_{k \ell}}
$$

with an appropriately chosen non-derivative DeWitt metric tensor $\mathcal{G}_{i j k \ell}$. This condition inspired by the theory of non-equilibrium systems - has a straightforward generalization in 
the presence of matter. When the theory is in detailed balance, the number of independent couplings in $\mathcal{V}$ reduces to the number of independent couplings in $\mathcal{W}$.

Note also that since the lapse function is the gauge field associated with the time reparametrization symmetry, it can be naturally restricted to be a function of time only; this leads to the projectable version of the theory. In that case, the potential term $\mathcal{V}$ is a local function of the Riemann tensor of the spatial metric $\gamma_{i j}$, and its covariant derivatives. It is also possible to relax the projectability condition, and allow $N$ to be a spacetime-dependent field; this yields the non-projectable version $[1-3,34,35]$. In the non-projectable theory, there is one additional ingredient that can be used to build the potential term $\mathcal{V}$ : the spatial vector field $\nabla_{i} N / N$.

\subsection{Anisotropic Weyl Transformations}

Under certain circumstances, we can impose additional gauge symmetries to further constrain the classical action of gravity with anisotropic scaling. When $z=D$, one can thus require invariance under a local version of the anisotropic scaling (1.1), which acts on the metric multiplet by anisotropic Weyl transformations

$$
N \mapsto e^{z \omega} N \quad N_{i} \mapsto e^{2 \omega} N_{i} \quad \gamma_{i j} \mapsto e^{2 \omega} \gamma_{i j}
$$

with an arbitrary local function $\omega(t, \mathbf{x})$. We denote the group of anisotropic Weyl transformations (2.7) with dynamical exponent $z$ by $\operatorname{Weyl}_{z}(M, \mathcal{F})$. Crucially, this group extends the group of foliation preserving diffeomorphisms into a semi-direct product $[1,18]$

$$
\operatorname{Weyl}_{z}(M, \mathcal{F}) \rtimes \operatorname{Diff}(M, \mathcal{F}) .
$$

Indeed, the commutator between an infinitesimal foliation-preserving diffeomorphism $\delta_{\left(f, \xi^{i}\right)}$ of (2.2) and an infinitesimal generator $\delta_{\omega}$ of the anisotropic Weyl transformation (2.7) yields another infinitesimal anisotropic Weyl transformation,

$$
\left[\delta_{\omega}, \delta_{\left(f, \xi^{i}\right)}\right]=\delta_{f \partial_{t} \omega+\xi^{i} \partial_{i} \omega}
$$

with the same fixed - but otherwise arbitrary - value of $z$. On the other hand, had we tried to extend $\operatorname{Diff}(M, \mathcal{F})$ into the full spacetime diffeomorphism group, the closure of the symmetries would have forced the relativistic scaling with $z=1$. Thus, anisotropic Weyl symmetry is only possible when we relax the spacetime diffeomorphism symmetry to the symmetries of the preferred foliation $\mathcal{F}$.

Since $\operatorname{Weyl}_{z}(M, \mathcal{F})$ acts on $N$ by a spacetime-dependent gauge transformation (2.7), $N$ itself must be a spacetime-dependent field, hence it cannot satisfy the projectability condition. This suggests that the natural environment for conformal gravity with anisotropic Weyl invariance is the non-projectable version of the theory. ${ }^{1}$

\footnotetext{
${ }^{1}$ One might consider restricting the Weyl invariant combination $\widetilde{N} \equiv N / \sqrt{\gamma}$ to be a function of time only [1]; we leave the study of such a "conformally projectable" theory outside of the scope of the present paper.
} 


\section{$2.2 z=2$ Conformal Gravity in $2+1$ Dimensions}

Insisting on the additional symmetries (2.7) implies that the coupling constant $\lambda$ must take a fixed value, $\lambda=1 / D$. We will refer to it as the "conformal value" of $\lambda$. In this paper, we will be mainly interested in the case of $D=2$, which requires $z=2$ and the unique kinetic term

$$
S_{K}=\frac{1}{\kappa^{2}} \int d t d^{2} \mathbf{x} \sqrt{\gamma} N\left(\hat{K}_{i j} \hat{K}^{i j}-\frac{1}{2} \hat{K}^{2}\right) .
$$

One can easily check that this term is indeed invariant under (2.7).

The potential term $\mathcal{V}$ is also strongly constrained by the condition of anisotropic Weyl invariance (2.7). In $D=2$, where the Riemann tensor of the spatial metric reduces to the Ricci scalar $\hat{R}$, there is only one term that can appear in $\mathcal{V}{ }^{2}$

$$
S_{\mathcal{V}}=\frac{1}{\kappa_{\mathcal{V}}^{2}} \int d t d^{2} \mathbf{x} \sqrt{\gamma} N\left\{\hat{R}+\frac{\nabla^{2} N}{N}-\left(\frac{\nabla N}{N}\right)^{2}\right\}^{2} .
$$

This term is also invariant under (2.7), but it does not satisfy the detailed balance condition: There is no local action that would yield this term as the sum of squares of its equations of motion. ${ }^{3}$ Thus, pure $z=2$ conformal gravity in $2+1$ dimensions with detailed balance has no potential term.

This conformal $z=2$ gravity in $2+1$ dimensions can be coupled to scalars $X^{a}(t, \mathbf{x})$. Anisotropic Weyl invariance of the classical action will be preserved when we assign scaling dimension zero to $X^{a}$. The kinetic term becomes

$$
S_{K}=\frac{1}{\kappa^{2}} \int d t d^{2} \mathbf{x} \sqrt{\gamma} N\left(\hat{K}_{i j} \hat{K}^{i j}-\frac{1}{2} \hat{K}^{2}\right)+\frac{1}{2} \int d t d^{2} \mathbf{x} \frac{\sqrt{\gamma}}{N}\left(\partial_{t} X^{a}-N^{i} \partial_{i} X^{a}\right)^{2} .
$$

Even under the condition of detailed balance, this coupled theory develops a nontrivial potential. There is a unique potential term compatible both with anisotropic conformal invariance and the detailed balance condition,

$$
S_{\mathcal{V}}=\int d t d^{2} \mathbf{x} \sqrt{\gamma} N\left\{\left(\nabla^{2} X^{a}\right)^{2}+\frac{\kappa^{2}}{2}\left(\partial_{i} X^{a} \partial_{j} X^{a}-\frac{1}{2} \gamma_{i j} \partial^{k} X^{a} \partial_{k} X^{a}\right)^{2}\right\} .
$$

This theory, of $z=2$ conformal gravity coupled to scalars and satisfying the detailed balance condition, first appeared in [1] as the worldvolume action of "membranes at quantum criticality," whose ground-state wavefunction on Riemann surface $\Sigma$ reproduces the partition function of the critical bosonic string on $\Sigma$. The Euclidean action in $D=2$ dimensions which yields (2.13) via the detailed balance construction is simply given by the action familiar from the critical string,

$$
\mathcal{W}=\frac{1}{2} \int d^{2} \mathbf{x} \sqrt{\gamma} \gamma^{i j} \partial_{i} X^{a} \partial_{j} X^{a}
$$

\footnotetext{
${ }^{2}$ Throughout the paper, we use the compact notation $\nabla^{2} N \equiv \nabla i \nabla^{i} N$ and $(\nabla N)^{2} \equiv \nabla_{i} N \nabla^{i} N$.

${ }^{3}$ However, as was discussed in [1], one can get $\mathcal{V} \sim R^{2}$ by squaring the equation of motion of a nonlocal action: the Polyakov conformal anomaly action $\int d^{2} \mathbf{x} \sqrt{\gamma} R \frac{1}{\nabla^{2}} R$.
} 
We recognize the first term in (2.13) as the square of the $X^{a}$ equation of motion, and the second term as the square of the energy-momentum tensor obtained from the $\gamma_{i j}$ variation of (2.14).

\section{Holography in Asymptotically Lifshitz Spacetimes}

The metric of the Lifshitz spacetime $\mathcal{M}$ in $D+2$ dimensions,

$$
d s^{2}=-r^{2 z} d t^{2}+r^{2} d \mathbf{x}^{2}+\frac{d r^{2}}{r^{2}}
$$

is designed so that its isometries match the expected conformal symmetries of Lifshitz field theory with dynamical exponent $z$. This geometry, and its various cousins, appears as a solution in several effective theories, such as the theory considered in [36] in which bulk Einstein gravity is coupled to a massive vector, and more recently also in a variety of constructions obtained from string theory [37-40].

In this section, we first discuss some general features of holography and asymptotic structure of Lifshitz spacetime, which are universal and independent of the precise model. Then, in Section 4 , we work - for specificity - in the effective bulk theory of relativistic gravity coupled to a massive vector, first without additional matter, and then coupled to bulk scalars. Even though our detailed results will depend of the chosen effective theory, we believe that our conclusions are largely universal and generalizable straightforwardly to other embeddings of Lifshitz spacetimes.

\subsection{Anisotropic Conformal Infinity}

The notion of conformal infinity plays a central role in general relativity $[17,41]$. It is constructed by mapping the original metric ${ }^{4} G_{\mu \nu}$ on a manifold $\mathcal{M}$ via a smooth conformal Weyl transformation to

$$
\widetilde{G}_{\mu \nu}=\Omega^{2}(x) G_{\mu \nu}
$$

such that the rescaled metric $\widetilde{G}_{\mu \nu}$ is extendible to a larger manifold $\widetilde{\mathcal{M}}$, which contains the closure $\overline{\mathcal{M}}$ of $\mathcal{M}$ as a closed submanifold. The idea is to define the conformal infinity of $\mathcal{M}$ to be the set $\overline{\mathcal{M}} \backslash \mathcal{M}$. The scaling factor $\Omega$ must extend to $\widetilde{\mathcal{M}}$ and satisfy certain regularity conditions at $\overline{\mathcal{M}} \backslash \mathcal{M}$ (the most essential being that it should have a single zero there and that its exterior derivative should be nonzero), but is otherwise arbitrary. A change from one permissible scaling factor to another is interpreted as a conformal transformation at $\overline{\mathcal{M}} \backslash \mathcal{M}$ : Hence, conformal infinity carries a naturally defined preferred conformal structure.

This notion of conformal infinity allows one to define precisely, and in a coordinateindependent way, the notion of an event horizon (and hence the notion of black holes), as the boundary of the causal past of the future infinity. Moreover, it allows us to define precisely the concept of spacetimes which "asymptotically approach" a chosen vacuum solution "at

\footnotetext{
${ }^{4}$ We are using Penrose's "abstract index" notation.
} 
infinity." In the example of anti-de Sitter spacetime, this picture is naturally compatible with the physical ideas of holography: The conformal infinity of $A d S$ is of codimension one, and carries the natural conformal structure induced from the asymptotic isometries of the bulk, just as predicted by the holographic dictionary.

In contrast, the intuition of holographic renormalization in Lifshitz and Schrödinger spacetimes clashes with this classic notion of conformal infinity as defined by Penrose: For the Lifshitz spacetime (3.1) with $z>1$, the relativistic conformal infinity is of dimension one for any $D$, and it does not inherit the conformal structure expected from the symmetries of nonrelativistic field theory in $D+1$ dimensions. To see that, it is useful to switch first to the radial coordinate $u=1 / r$, which stays finite as we approach the naive infinity at $r \rightarrow \infty$, with the metric now

$$
d s^{2}=-\frac{d t^{2}}{u^{2 z}}+\frac{d \mathbf{x}^{2}+d u^{2}}{u^{2}} .
$$

For $z>1$, the $d t^{2}$ term is the most divergent one as we take $u \rightarrow 0$. In order to make the rescaled metric finite, we would like to use $\Omega=u^{z}$. However, this choice of $\Omega$ does not have a simple zero at $u=0$ in this coordinate system. In order to fix this, we change coordinates once again, to $w=u^{z}$. The metric becomes

$$
d s^{2}=-\frac{d t^{2}}{w^{2}}+\frac{d \mathbf{x}^{2}}{w^{2 / z}}+\frac{d w^{2}}{z^{2} w^{2}} .
$$

We can now use $\Omega=w$ to rescale the metric, but the resulting geometry

$$
\widetilde{d s}^{2}=-d t^{2}+\frac{1}{z^{2}} d w^{2}+w^{2(1-1 / z)} d \mathbf{x}^{2}
$$

is degenerate at the purported infinity $w=0$ when $z \neq 1$. As a consequence of this rather pathological behavior of the standard notion of conformal infinity of the Lifshitz spacetime, it is a priori unclear how to perform holographic renormalization, and even how to define precisely what we mean by black holes in the bulk.

This tension has been remedied [18], for spacetimes carrying the additional structure of an asymptotic foliation, by generalizing Penrose's notion of conformal infinity to reflect the asymptotic anisotropy permitted by the foliation. The basic idea is simple: When $\mathcal{M}$ carries a preferred foliation at least near infinity, we can use the anisotropic Weyl transformation (2.7), instead of the relativistic rescaling (3.2), to map $\mathcal{M}$ inside a larger manifold $\widetilde{\mathcal{M}}$ such that $\overline{\mathcal{M}} \subset \widetilde{\mathcal{M}}$. Even in this case, the rescaling factor $\Omega=e^{\omega}$ must satisfy regularity conditions at $\overline{\mathcal{M}} \backslash \mathcal{M}$. In particular, $\Omega$ must have a simple zero there. With a judiciously chosen value of $z$, the anisotropic conformal infinity $\overline{\mathcal{M}} \backslash \mathcal{M}$ can be of codimension one. Moreover, it naturally inherits a preferred "anisotropic conformal structure," with conformal transformations given by those foliation-preserving diffeomorphisms that preserve the boundary metric up to an anisotropic Weyl rescaling.

Both Lifshitz and Schrödinger spacetimes belong to this class of asymptotically foliated geometries, and the resulting notion of anisotropic conformal infinity matches the intuitive expectations from holography [18]. In the case of the Lifshitz spacetime (3.1), we start 
again with the metric as given in (3.3). We interpret this geometry as carrying a natural codimension-one foliation by leaves of constant $t$, at least near $u \rightarrow 0$. As we saw in Section 2.1, this additional structure of an asymptotic foliation gives us the additional freedom to use anisotropic Weyl transformations 2.7 without violating the symmetries. Choosing the rescaling factor

$$
\Omega=u
$$

and applying the anisotropic Weyl transformation (2.7) maps the Lifshitz metric in the asymptotic regime of $u \rightarrow 0$ to the flat metric,

$$
\widetilde{d s}^{2}=-d t^{2}+d \mathbf{x}^{2}+d u^{2}
$$

$u$ can now be analytically extended from $u>0$ to all real values. The anisotropic conformal infinity of the $(D+2)$-dimensional Lifshitz spacetime is at $u=0$. Topologically, it is $\mathbf{R}^{D+1}$, and very similar to the conformal infinity of the Poincaré patch of $A d S_{D+2}$. However, even though the induced metric on anisotropic conformal infinity at $u=0$ in (3.7) looks naively relativistic, one must remember that its natural symmetries are not relativistic: This conformal infinity carries a preferred foliation by leaves of constant $t$, and a natural anisotropic conformal structure characterized by dynamical exponent $z$. The natural symmetries are given by those foliation-preserving diffeomorphisms that preserve the metric up to an anisotropic Weyl transformation [18]. In addition to the spatial rotations and spacetime translations of $\mathbf{R}^{D+1}$, one can easily check that this symmetry group contains also the anisotropic scaling transformations (1.1). Thus, the conformal structure of anisotropic conformal infinity nicely matches the expected conformal symmetries of the dual field theory.

\subsection{Asymptotically Lifshitz Spacetimes}

Equipped with the notion of anisotropic conformal infinity of spacetime, we can now give a precise definition of spacetime geometries that are "asymptotically Lifshitz." Simply put, given a value of $z$, a spacetime is said to be asymptotically Lifshitz if it exhibits the same anisotropic conformal infinity as the Lifshitz spacetime for that value of dynamical exponent $z$. This definition follows the logic that leads to the notions of asymptotic flatness and asymptotic $\operatorname{AdS}[17,41]$, and extends such notions naturally to the case of anisotropic scaling.

As a part of their definition, the spacetimes which are asymptotically Lifshitz must carry an asymptotic foliation structure near their anisotropic conformal infinity. In the context of holographic renormalization, this condition translates into an important restriction on the form of the vielbein fall-off,

$$
\frac{e_{i}^{0}}{r^{z}} \rightarrow 0 \quad \text { as } r \rightarrow \infty
$$

This provides an answer to a question discussed in [42]: Our definition of asymptotically Lifshitz spacetimes using the notion of anisotropic conformal infinity requires that the sources for the energy flux vanish. ${ }^{5}$

\footnotetext{
${ }^{5}$ More precisely, it would be sufficient to impose $\left(\partial_{i} e_{j}^{0}-\partial_{j} e_{i}^{0}\right) / r^{z} \rightarrow 0$ at infinity, a constraint which also
} 
With the definition of "asymptotically Lifshitz" at hand, it is now possible to define precisely black holes and their event horizons in Lifshitz spacetimes, by referring to the properties of the anisotropic conformal infinity of spacetime just as in the more traditional spacetimes which have codimension-one isotropic conformal infinity. We refer the reader to [18] for additional results, and to Appendix A.6 for a summary of the asymptotic behavior of fields in the asymptotically Lifshitz spacetimes relevant for the rest of this paper.

\section{Holographic Renormalization in Asymptotically Lifshitz Spacetimes}

Holographic duality in asymptotically $A d S$ spacetimes $^{6}$ - or, by logical extension, in asymptotically Lifshitz spacetimes - relates the partition function of a bulk gravity system with Dirichlet boundary conditions at conformal infinity to the generating function of correlators in the appropriate dual quantum field theory. At low energies and to leading order, this correspondence gives the connected generating functional $W$ with sources $f^{(0)}$ on the field theory side, in terms of the on-shell bulk gravity action evaluated with Dirichlet boundary conditions given by $f^{(0)}$ :

$$
W\left[f^{(0)}\right]=-S_{\text {on-shell }}\left[f^{(0)}\right]
$$

Both sides of this correspondence are divergent: Standard ultraviolet divergences appear on the field theory side, and they require conventional renormalization. This behavior is matched on the gravity side, where the divergences are infrared effects, due to the scales that diverge as we approach the spacetime boundary. Holographic renormalization [19,45-49] (for reviews, see $[26,50,51])$ is the technology designed to perform the subtraction of infinities on the gravity side, in the form of divergent boundary terms in the on-shell action, and to make precise sense of (4.1).

Recent papers $[42,52,53]$ have performed various steps of holographic renormalization in Lifshitz spacetime at the non-linear level, and our paper builds on the results established there. Since we choose for our analysis the Hamiltonian approach to holographic renormalization $[54,55]$, our treatment is closest to that of $[42]$.

\subsection{Hamiltonian Approach to Holographic Renormalization}

The original analysis of holographic renormalization relied on properties of asymptotic expansions near the conformal infinity of spacetime [56-58]. The Hamiltonian approach of $[54,55]$ aspires to give a somewhat more covariant picture, and the results of the earlier asymptotic expansion approach can be reproduced from it [54]. Either way, we start by choosing a radial coordinate, $r$, in some neighborhood of the anisotropic conformal infinity of the Lifshitz spacetime $\mathcal{M}$, such that the hypersurfaces of constant $r$ are diffeomorphic to the boundary

emerges naturally in the vielbein formulation of gravity with anisotropic scaling. In this paper, we impose the stronger condition (3.8).

${ }^{6}$ For a pedagogical introduction, see the TASI lectures [43] and [44]. 
$\partial \mathcal{M}$, and they equip $\mathcal{M}$ near $\partial \mathcal{M}$ with a codimension-one foliation structure. ${ }^{7}$ This foliation should not be confused with the preferred folation of the anisotropic conformal boundary by leaves of constant $t$ - the asymptotic regime of our spacetime carries a nested foliation structure, with leaves of constant radial coordinate $r$ further foliated by leaves of constant $t$.

Our starting point is the theory of bulk gravity in $3+1$ dimensions $^{8}$ with negative $\Lambda$, coupled to some matter $\Phi$ to be specified later. The action is given by

$$
S_{\text {bulk }}=\frac{1}{16 \pi G_{4}} \int_{\mathcal{M}} d t d^{2} \mathbf{x} d r \sqrt{-G}(\mathcal{R}-2 \Lambda)+\frac{1}{8 \pi G_{4}} \int_{\partial \mathcal{M}} d t d^{2} \mathbf{x} \sqrt{-g} K+S_{\text {matter }}[\Phi, G] .
$$

We will work throughout in the radial gauge, setting the radial lapse to $1 / r$ and the radial shift to zero, in some neighborhood of the boundary (see Appendix A for a detailed summary of our notation and conventions).

Our task is to evaluate the on-shell action as a functional of the boudary fields, and perform the corresponding renormalization. Because of the infinite volume of Lifshitz space, the on-shell action diverges, and must first be regularized by inserting a cutoff at finite volume and indentifying terms that diverge in the asymptotic expansion in the cutoff, and then renormalized by and introducing appropriate counterterms to eliminate the divergences. The on-shell action is regulated by cutting the bulk spacetime off at some value $r<\infty$ of the radial coordinate. If $\mathcal{M}_{r}$ is the cut-off manifold, its boundary $\partial \mathcal{M}_{r}$ represents a regulated boundary of spacetime. The on-shell action is a function of the regulator $r$, and the boundary fields which include the metric multiplet $N, N_{i}, \gamma_{i j}$ plus all sources associated with the bulk matter $\Phi$, which we collectively denote by $\phi$. From now on, we simply denote the on-shell

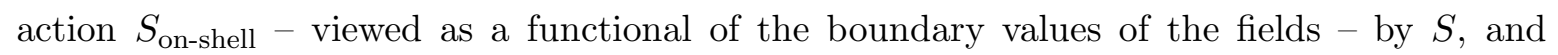
parametrize it as

$$
S=\frac{1}{16 \pi G_{4}} \int d t d^{2} \mathbf{x} \sqrt{\gamma} N \mathcal{L}
$$

Since the on-shell action $S$ is a function of $r$ and the boundary values of the fields, we can naturally interpret it as a solution to the Hamilton-Jacobi equation, regarding $r$ as the evolution parameter. This is the starting point for the Hamiltonian approach to holographic renormalization. The Hamilton-Jacobi theory implies that the first variation of the on-shell action with respect to the boundary fields gives the conjugate momenta. In the holographic dictionary, the boundary fields serve as sources, and their conjugate momenta are thus directly related to the one-point functions of the operators conjugate to the sources.

A convenient way of computing the divergent part of $\mathcal{L}$ is to organize the terms with respect to their scaling with $r$. More precisely, we define the dilatation operator by

$$
\delta_{\mathcal{D}}=\int_{\partial \mathcal{M}_{r}} d t d^{2} \mathbf{x}\left(z N \frac{\delta}{\delta N}+2 N_{i} \frac{\delta}{\delta N_{i}}+2 \gamma_{i j} \frac{\delta}{\delta \gamma_{i j}}-\sum_{\phi} \Delta_{\phi} \phi \frac{\delta}{\delta \phi},\right)
$$

\footnotetext{
${ }^{7}$ In our conventions, $\partial \mathcal{M}$ is at $r=\infty$. The choice of $u=1 / r$ instead of $r$ as a coordinate near $\partial \mathcal{M}$ would be more appropriate, since $u$ is finite through $\partial \mathcal{M}$. In this section, we leave this more rigorous coordinate choice implicit.

${ }^{8}$ The case of general $D$ and $z$ is discussed in Appendix A.
} 
where $\Delta_{\phi}$ collectively denotes the asymptotic decay exponents of the bulk matter fields $\Phi$. Quantities of interest can then be decomposed into a sum of terms with definite scaling dimension under $\delta_{\mathcal{D}}$. For example, the object of our central interest, $\mathcal{L}$, can be expanded as

$$
\mathcal{L}=\sum_{\Delta} \mathcal{L}^{(\Delta)}+\widetilde{\mathcal{L}}^{(z+2)} \log r
$$

Throughout this paper, superscripts in parentheses on any object $\mathcal{O}$ always denote the scaling dimension in the decomposition of $\mathcal{O}$ as a sum of terms of definite engineering scaling dimensions. For example, $T_{A B}^{(0)}$ is the constant part of the stress tensor, and $R^{(2)}$ is the dimension-two part of the scalar curvature.

The individual terms of the expansion (4.5) satisfy

$$
\delta_{\mathcal{D}} \mathcal{L}^{(\Delta)}=-\Delta \mathcal{L}^{(\Delta)} \quad \text { for } \quad \Delta \neq z+2
$$

When $\Delta=z+2$, the scaling behavior is anomalous,

$$
\delta_{\mathcal{D}} \mathcal{L}^{(z+2)}=-(z+2) \mathcal{L}^{(z+2)}+\widetilde{\mathcal{L}}^{(z+2)},
$$

with the inhomogeneous term satisfying

$$
\delta_{\mathcal{D}} \widetilde{\mathcal{L}}^{(z+2)}=-(z+2) \widetilde{\mathcal{L}}^{(z+2)} .
$$

This logarithmic term in (4.5) reflects the possibility of an anisotropic Weyl anomaly.

The dynamical equations for the divergent part of $\mathcal{L}$ are determined as follows. Since the on-shell action satisfies the Hamilton-Jacobi equation, its radial derivative is determined in terms of the Hamiltonian. Because in our case the fields have fixed asymptotic behavior (see Appendix A.6), in the asymptotic region the radial derivative is equivalent to the anisotropic scaling operator,

$$
r \frac{d}{d r} \approx \delta_{\mathcal{D}}
$$

The Hamilton-Jacobi equation then relates the action of $\delta_{\mathcal{D}}$ on the on-shell action to the Hamiltonian of the system. In our case, with relativistic gravity in the bulk, the equation of motion for the radial lapse gives the Hamiltonian constraint. Using the bulk equations of motion, one obtains a first-order differential equation for $\mathcal{L}$ in terms of the boundary values of the fields that can be solved iteratively in the expansion in eigenmodes of $\delta_{\mathcal{D}}$.

Equivalently, one can expand the Hamiltonian constraint in eigenmodes of $\delta_{\mathcal{D}}$. The structure of these equations allows for the momentum modes to be obtained recursively in terms of the boundary data. In this method, the dilatation operator acting on the onshell action gives an expression linear in the canonical momenta, so that the values for the momenta obtained recursively from the Hamiltonian constraint give rise directly to the desired expression on-shell action. The resulting on-shell action will have divergent pieces that can be expressed as local functionals of the boundary data. These pieces can be subtracted, leading to the finite renormalized on-shell action.

Further technical details of the procedure for determining the coefficients $\mathcal{L}^{(\Delta)}$ and $\widetilde{\mathcal{L}}^{(z+2)}$ are summarized in Appendix B. 


\subsection{Bulk Gravity with a Massive Vector}

We begin with the theory of relativistic bulk gravity in $3+1$ dimensions, coupled to a bulk massive vector field $\mathcal{A}_{\mu}$. The action is

$$
\begin{gathered}
S_{\text {bulk }}=\frac{1}{16 \pi G_{4}} \int_{\mathcal{M}} d t d^{2} \mathbf{x} d r \sqrt{-G}\left(\mathcal{R}-2 \Lambda-\frac{1}{4} F_{\mu \nu} F^{\mu \nu}-\frac{1}{2} m^{2} \mathcal{A}_{\mu} \mathcal{A}^{\mu}\right) \\
+\frac{1}{8 \pi G_{4}} \int_{\partial \mathcal{M}} d t d^{2} \mathbf{x} \sqrt{-g} K .
\end{gathered}
$$

As summarized in Appendix A, this theory has the Lifshitz spacetime as a solution. In this theory, the boundary data we can specify reduce to the metric multiplet $\left(N, N_{i}, \gamma_{i j}\right)$ - or, alternatively, their vielbein counterparts (see Appendix A.3) - and a scalar source $\psi$ for the massive vector.

Although our main interest will be in $z=2$, we start by considering general $z$. If we set $\psi=0$, the terms that will give rise to divergent contributions in the on-shell action for $z<4$ are $\mathcal{L}^{(0)}, \mathcal{L}^{(2)}, \mathcal{L}^{(2 z)}$, and $\mathcal{L}^{(4)}$. The holographic renormalization equations, found in in [42] (and reviewed in Appendix B), take the form

$$
\begin{aligned}
\mathcal{L}^{(0)} & =2(z+1), \\
z \mathcal{L}^{(2)} & =R^{(2)}-\frac{1}{4}\left(F_{A B} F^{A B}\right)^{(2)}, \\
(2-z) \mathcal{L}^{(2 z)} & =R^{(2 z)}+\frac{1}{2 m^{2}}\left(\left(\nabla_{A} \pi^{A}\right)^{(z)}\right)^{2} \\
(z-2) \mathcal{L}^{(4)} & =K_{A B}^{(2)} \pi^{A B(2)}+\frac{1}{2} \pi^{A(2)} \pi_{A}{ }^{(2)} .
\end{aligned}
$$

With some effort these can be computed in terms of the boundary metric multiplet $\left(N, N_{i}, \gamma_{i j}\right)$, giving (up to total derivatives)

$$
\begin{aligned}
\mathcal{L}^{(0)}= & 2(z+1) \\
z \mathcal{L}^{(2)}= & \hat{R}+\frac{\alpha^{2}}{2}\left(\frac{\nabla N}{N}\right)^{2} \\
(2-z) \mathcal{L}^{(2 z)}= & \hat{K}_{i j} \hat{K}^{i j}+\frac{z-3}{2} \hat{K}^{2}, \\
(2-z) \mathcal{L}^{(4)}= & \frac{z-2}{2 z^{4}(z+1)\left(z-2+\beta_{z}\right)^{2}}\left\{-4 z\left(z-6+\beta_{z}\right)\left(\frac{\nabla^{2} N}{N}\right)^{2}\right. \\
& +\left(12+36 z-11 z^{2}-2 z^{3}+5 z^{4}+\beta_{z}\left(z^{3}-7 z-2\right)\right)\left[\left(\frac{\nabla N}{N}\right)^{2}\right]^{2} \\
& -2 z\left(36-4 z-7 z^{2}+5 z^{3}+\beta_{z}\left(z^{2}-z-6\right)\right) \frac{\nabla^{2} N}{N}\left(\frac{\nabla N}{N}\right)^{2} \\
& \left.\quad+\left(z-6+\beta_{z}\right)\left[4 z^{2}\left(\frac{\nabla N}{N}\right)^{2} \hat{R}-4 z^{2} \frac{\nabla^{2} N}{N} \hat{R}-z^{3} \hat{R}^{2}\right]\right\}
\end{aligned}
$$

When $z=2$ is approached, the divergent terms of dimension four become logarithmic, and the residue of the $\Delta=4$ (or $\Delta=2 z$ ) terms at the $z=2$ pole give rise to $\widetilde{\mathcal{L}}^{(4)}$. Specifically, 
we get

$$
\widetilde{\mathcal{L}}^{(4)}=\lim _{z \rightarrow 2}\left[(z-2) \mathcal{L}^{(4)}+(2-z) \mathcal{L}^{(2 z)}\right] .
$$

With this substitution, the $z=2$ divergent terms in the on-shell action are

$$
\begin{aligned}
\mathcal{L}^{(0)} & =2(z+1)=6, \\
\mathcal{L}^{(2)} & =\frac{1}{2} \hat{R}+\frac{1}{4}\left(\frac{\nabla N}{N}\right)^{2}, \\
\widetilde{\mathcal{L}}^{(4)} & =\hat{K}_{i j} \hat{K}^{i j}-\frac{1}{2} \hat{K}^{2} .
\end{aligned}
$$

The coefficient $\widetilde{\mathcal{L}}^{(4)}$ of the logarithmic divergence can be recognized as the unique kinetic term (2.10) for Lifshitz gravity with local conformal invariance in $2+1$ dimensions, invariant under the $z=2$ anisotropic Weyl transformations (2.7). This is one of the central results of this paper.

The expression for the counterterms has no potential term - i.e., the only derivatives that appear in the counterterm are the time derivatives. This is in spite of the fact that there exists a term with spatial derivatives, invariant under the local $z=2$ anisotropic Weyl transformations,

$$
\int d t d^{2} \mathbf{x} \sqrt{g} N\left\{\hat{R}+\frac{\nabla^{2} N}{N}-\left(\frac{\nabla N}{N}\right)^{2}\right\}^{2},
$$

which is not a total derivative.

It is surprising, at least at first sight, that such a potential term is not generated in the logarithmic counterterm of holographic renormalization in Lifshitz space. Indeed, as we show in Appendix Q, this term (4.23) represents a non-trivial cohomology class appropriate to appear as an anomaly. What would be a minimal generalization of our holographic setup, which would generate such a term in the anomaly? One might suspect that a different dynamical embedding of the Lifshitz space may perhaps produce a more general set of holographic counterterms, allowing (4.23) to appear. Even in the embedding considered here, we have not turned on the most general sources in the boundary, and one can ask whether allowing nonzero $\psi$ generates new counterterms. However, a detailed calculation (see Appendix B) reveals that turning on $\psi$ also preserves detailed balance, and does not lead to the appearance of the second independent counterterm (4.23).

\subsection{Gravity with a Massive Vector Coupled to Bulk Scalars}

In order to probe further the structure of holographic counterterms in Lifshitz spacetime, it is useful to add additional matter fields in the bulk theory. The holographic renormalization procedure can be easily repeated with the inclusion of scalar fields in the bulk. We will see that for a marginal scalar at $z=2$, there is a new logarithmically divergent counterterm, giving rise to a new, nongravitational contribution to the anisotropic Weyl anomaly. However, 
we will see that this new counterterm also satisfies the detailed balance condition: Even in the presence of the bulk scalars, the second gravitational counterterm (4.23) - which violates detailed balance - is not generated.

The bulk scalar action takes the standard relativistic form

$$
S_{\text {bulk, } X}=-\frac{1}{2} \int_{\mathcal{M}} d^{d+1} x \sqrt{-G}\left(G^{\mu \nu} \partial_{\mu} X^{a} \partial_{\nu} X^{a}+\mu^{2} X^{a} X^{a}\right) .
$$

In this section, we set $d=3$, and again follow the procedure of [42], with appropriate modifications to include the scalar fields. The holographic renormalization equations of [42] now become

$$
(z+2-\Delta) \mathcal{L}^{(\Delta)}=\widetilde{\mathcal{Q}}^{(\Delta)}+\widetilde{\mathcal{S}}^{(\Delta)}
$$

where the quadratic and source terms $\mathcal{Q}$ and $\mathcal{S}$ are modified to

$$
\widetilde{\mathcal{Q}}^{(\Delta)}=\mathcal{Q}^{(\Delta)}+8 \pi G_{4}\left(\widetilde{\pi}^{a(\Delta / 2)}\right)^{2}+16 \pi G_{4} \sum_{s<\Delta / 2 ; s \neq \widetilde{\Delta}_{-}}\left(\widetilde{\pi}^{a(s)} \widetilde{\pi}^{a(\Delta-s)}\right)
$$

and

$$
\widetilde{\mathcal{S}}=\mathcal{S}-8 \pi G_{4}\left(\partial_{\alpha} X^{a} \partial^{\alpha} X^{a}+\mu^{2} X^{a} X^{a}\right)
$$

In this expression, $\widetilde{\pi}^{a}=r \partial_{r} X^{a}$ is the scalar momentum and the scalars fall of asymptotically as $r^{-\widetilde{\Delta}_{-}}$, where

$$
\mu^{2}=\widetilde{\Delta}_{-}\left(\widetilde{\Delta}_{-}-2-z\right)
$$

The additional source terms only contribute at orders $\Delta=2 \widetilde{\Delta}_{-}, 2+2 \widetilde{\Delta}_{-}$and $2 z+2 \widetilde{\Delta}_{-}$:

$$
\begin{aligned}
\widetilde{\mathcal{S}}^{\left(2 \widetilde{\Delta}_{-}\right)} & =-8 \pi G_{4} \mu^{2} X^{a} X^{a} \\
\widetilde{\mathcal{S}}^{\left(2+2 \widetilde{\Delta}_{-}\right)} & =-\left[8 \pi G_{4} \partial_{\alpha} X^{a} \partial^{\alpha} X^{a}\right]^{\left(2+2 \widetilde{\Delta}_{-}\right)}=-8 \pi G_{4} \partial_{i} X^{a} \partial^{i} X^{a} \\
\widetilde{\mathcal{S}}^{\left(2 z+2 \widetilde{\Delta}_{-}\right)} & =-\left[8 \pi G_{4} \partial_{\alpha} X^{a} \partial^{\alpha} X^{a}\right]^{\left(2 z+2 \widetilde{\Delta}_{-}\right)}=\frac{8 \pi G_{4}}{N^{2}}\left(\partial_{t} X^{a}-N^{i} \partial_{i} X^{a}\right)^{2}
\end{aligned}
$$

We now specialize to the case of a marginal scalar, that is, a scalar which has $\widetilde{\Delta}_{-}=0$. Note that this also means that the scalar is massless since $\mu^{2}=\widetilde{\Delta}_{-}\left(\widetilde{\Delta}_{-}-2-z\right)=0$. We are interested in calculating the contribution to the anisotropic Weyl anomaly in the case $z=2$. The divergent pieces of the on-shell action that appear at orders $\Delta=2+2 \widetilde{\Delta}_{-}$and $\Delta=2 z+2 \widetilde{\Delta}_{-}$are straightforward to calculate as they only receive contributions from the source terms,

$$
\begin{aligned}
\left(z-2 \widetilde{\Delta}_{-}\right) \mathcal{L}^{\left(2+2 \widetilde{\Delta}_{-}\right)} & =-8 \pi G_{4} \partial_{i} X^{a} \partial^{i} X^{a} \\
\left(2-z-2 \widetilde{\Delta}_{-}\right) \mathcal{L}^{\left(2 z+2 \widetilde{\Delta}_{-}\right)} & =\frac{8 \pi G_{4}}{N^{2}}\left(\partial_{t} X^{a}-N^{i} \partial_{i} X^{a}\right)^{2}
\end{aligned}
$$

By taking the functional derivative of this term in the on-shell action with respect to the metric, the contribution to the boundary stress energy tensor can be calculated. For example, 
for $\Delta=2+2 \widetilde{\Delta}_{-}$

$$
\begin{aligned}
\left(z-2 \widetilde{\Delta}_{-}\right) T_{00}^{\left(2+2 \widetilde{\Delta}_{-}\right)} & =-8 \pi G_{4} \partial_{i} X^{a} \partial^{i} X^{a}, \\
\left(z-2 \widetilde{\Delta}_{-}\right) T_{I J}^{\left(2+2 \widetilde{\Delta}_{-}\right)} & =-16 \pi G_{4} \partial_{I} X^{a} \partial_{J} X^{a}+8 \pi G_{4} \partial_{i} X^{a} \partial^{i} X^{a} \delta_{I J}, \\
\left(z-2 \widetilde{\Delta}_{-}\right) T_{0 I}^{\left(3-z+2 \widetilde{\Delta}_{-}\right)} & =0 .
\end{aligned}
$$

In addition, by taking the functional derivative with respect to the scalar, the boundary scalar momentum can be calculated, via

$$
\tilde{\pi}^{a}=-\frac{1}{N \sqrt{\gamma}} \frac{\delta S}{\delta X^{a}}
$$

For example, one gets

$$
\left(z-2 \widetilde{\Delta}_{-}\right) \widetilde{\pi}^{a\left(2+\widetilde{\Delta}_{-}\right)}=-\frac{1}{N} \nabla^{i}\left(N \nabla_{i} X^{a}\right)=-\nabla^{2} X^{a}-\frac{\nabla^{i} N \nabla_{i} X^{a}}{N} .
$$

The higher order counterterms are more involved because they receive contributions from the quadratic piece. For example,

$$
\begin{aligned}
\left(z-\Delta_{-}\right. & \left.-2 \widetilde{\Delta}_{-}\right) \mathcal{L}^{\left(2+\Delta_{-}+2 \widetilde{\Delta}_{-}\right)}=K_{A B}^{\left(\Delta_{-}\right)} T^{A B\left(2+2 \widetilde{\Delta}_{-}\right)} \\
& =-\frac{\alpha \psi}{2(z+1)}\left[z\left(3 z-\Delta_{-}\right) T^{00\left(2+2 \widetilde{\Delta}_{-}\right)}+z\left(2 z-1-\Delta_{-}\right) T_{I}^{I\left(2+2 \widetilde{\Delta}_{-}\right)}\right] \\
& =-\frac{\alpha \psi}{2(z+1)}\left[z\left(3 z-\Delta_{-}\right) T^{00\left(2+2 \widetilde{\Delta}_{-}\right)}\right]
\end{aligned}
$$

using the fact that $T_{I}^{I^{\left(2+2 \widetilde{\Delta}_{-}\right)}}=0$, as calculated above. Note that for $z=2$ this becomes $\mathcal{L}^{\left(2+\Delta_{-}+2 \widetilde{\Delta}_{-}\right)}=-\psi T^{00\left(2+2 \widetilde{\Delta}_{-}\right)}$. The calculation of this term is useful even when the source for the massive vector $\psi$ is set to zero. This is because we can determine $\pi_{\psi}^{\left(2+2 \widetilde{\Delta}_{-}\right)}$by taking the functional derivative with respect to $\psi$ :

$$
\left(z-\Delta_{-}-2 \widetilde{\Delta}_{-}\right) \pi_{\psi}^{\left(2+2 \widetilde{\Delta}_{-}\right)}=\frac{\alpha}{2(z+1)}\left[z\left(3 z-\Delta_{-}\right) T^{00\left(2+2 \widetilde{\Delta}_{-}\right)}\right] .
$$

The following terms also receive contributions from the quadratic piece:

$$
\begin{aligned}
& \left(z-2-2 \widetilde{\Delta}_{-}\right) \mathcal{L}^{\left(4+2 \widetilde{\Delta}_{-}\right)}=2 K_{A B}^{(2)} T^{A B\left(2+2 \widetilde{\Delta}_{-}\right)}+\pi_{A}^{(2)} \pi^{A\left(2+2 \widetilde{\Delta}_{-}\right)}+8 \pi G_{4}\left(\widetilde{\pi}^{a\left(2+\widetilde{\Delta}_{-}\right)}\right)^{2}, \\
& \left(z-2-4 \widetilde{\Delta}_{-}\right) \mathcal{L}^{\left(4+4 \widetilde{\Delta}_{-}\right)}=K_{A B}^{\left(2+2 \widetilde{\Delta}_{-}\right)} T^{A B\left(2+2 \widetilde{\Delta}_{-}\right)}+\frac{1}{2} \pi_{A}^{\left(2+2 \widetilde{\Delta}_{-}\right)} \pi^{A\left(2+2 \widetilde{\Delta}_{-}\right)} .
\end{aligned}
$$

These are the terms that will contribute to the scaling anomaly when $z=2$. After a lengthy calculation of the right hand sides for $z=2$, the following result is obtained (up to total derivatives):

$$
\begin{aligned}
& \left(z-2-2 \widetilde{\Delta}_{-}\right) \mathcal{L}^{\left(4+2 \widetilde{\Delta}_{-}\right)}=2 \pi G_{4}\left(\Delta X^{a}\right)^{2} \\
& \left(z-2-4 \widetilde{\Delta}_{-}\right) \mathcal{L}^{\left(4+4 \widetilde{\Delta}_{-}\right)}=\frac{1}{4} T_{I J}^{\left(2+2 \widetilde{\Delta}_{-}\right)} T^{I J\left(2+2 \widetilde{\Delta}_{-}\right)},
\end{aligned}
$$




$$
=16 \pi^{2} G_{4}^{2}\left(\partial_{i} X^{a} \partial_{j} X^{a} \partial^{i} X^{b} \partial^{j} X^{b}-\frac{1}{2}\left(\partial_{i} X^{a} \partial^{i} X^{a}\right)^{2}\right) .
$$

By combining all these results, the contribution of the massless scalars to the logarithmically divergent counterterm when $z=2$ is (by equation $(\underline{B} .10)$ ):

$$
\begin{aligned}
& \widetilde{\mathcal{L}}_{X}^{(4)}= \lim _{z \rightarrow 2}\left((2-z) \mathcal{L}^{\left(2 z+2 \widetilde{\Delta}_{-}\right)}+(z-2) \mathcal{L}^{\left(4+2 \widetilde{\Delta}_{-}\right)}+(z-2) \mathcal{L}^{\left(4+4 \widetilde{\Delta}_{-}\right)}\right) \\
&=\frac{8 \pi G_{4}}{N^{2}}\left(\partial_{t} X^{a}-N^{i} \partial_{i} X^{a}\right)^{2}+2 \pi G_{4}\left(\nabla^{2} X^{a}\right)^{2} \\
& \quad+16 \pi^{2} G_{4}^{2}\left(\partial_{i} X^{a} \partial_{j} X^{a} \partial^{i} X^{b} \partial^{j} X^{b}-\frac{1}{2}\left(\partial_{i} X^{a} \partial^{i} X^{a}\right)^{2}\right) .
\end{aligned}
$$

Together with the gravitational counterterms from the previous section, the total counterterm action for $z=2$ is given by

$$
\begin{aligned}
S_{c t}=-\int_{\partial \mathcal{M}} d t & d^{2} \mathbf{x} \sqrt{\gamma} N\left\{\frac{1}{16 \pi G_{4}}\left[6+\frac{1}{2} \hat{R}+\frac{1}{4}\left(\frac{\nabla N}{N}\right)^{2}\right]-\frac{1}{4} \partial_{i} X^{a} \partial^{i} X^{a}\right. \\
-\log \epsilon & {\left[\frac{1}{16 \pi G_{4}}\left(\hat{K}_{i j} \hat{K}^{i j}-\frac{1}{2} \hat{K}^{2}\right)+\frac{1}{2 N^{2}}\left(\partial_{t} X^{a}-N^{i} \partial_{i} X^{a}\right)^{2}+\frac{1}{8}\left(\nabla^{2} X^{a}\right)^{2}\right.} \\
+ & \left.\left.\pi G_{4}\left(\partial_{i} X^{a} \partial_{j} X^{a} \partial^{i} X^{b} \partial^{j} X^{b}-\frac{1}{2}\left(\partial_{i} X^{a} \partial^{i} X^{a}\right)^{2}\right)\right]\right\} .
\end{aligned}
$$

Interestingly, this logarithmically divergent counterterm takes the form identical to the action written down in [1], describing the coupling of $z=2$ gravity and $z=2$ Lifshitz matter in $2+1$ dimensions. This action is invariant under $z=2$ anisotropic Weyl transformations, with the scalars transforming with weight zero, and satisfies the detailed balance condition. As a result, it was shown in [1] that the ground-state wavefunction of this membrane action on a spatial surface $\Sigma$ is given by the bosonic string partition function on $\Sigma$. We see that the property of detailed balance, satisfied by the logarithmic counterterms in the absence of extra matter, persists in the presence of the marginal scalar fields.

Two additional comments are worth making:

(1) The relative sign between the potential terms and the kinetic term in the logarithmic counterterm is opposite to the sign one would expect from the action of a unitary theory with $z=2$ scaling in real time. This is not very surprising, and corresponds to the fact already appreciated in the relativistic case: The holographic counterterms do not have to reproduce the action of a unitary theory, as is clear from the appearance of the higherderivative conformal gravity action in the holographic counterterms in $A d S_{5}$.

(2) In the classical theories with Lifshitz scaling, the coupling constants in front of the individual contributions to the potential term are not related by any symmetry to the kinetic terms. Therefore, they represent classically marginal couplings. In the structure of our counterterms, we find this freedom realized only partially: A uniform overall rescaling of all the couplings in the potential can be accomplished by a shift in $r$, but it appears that the interaction with the bulk relativistic system eliminates the apparent freedom of the relative rescaling between different contributions to the potential from species unrelated by any symmetry in the boundary theory. This mechanism deserves further study. 


\subsection{Explaining Detailed Balance}

Now that we have accumulated some evidence suggesting that the appearance of the detailed balance condition in the structure of the counterterms is rather generic, it would be desirable to obtain a more systematic explanation of this fact. It would be interesting to see why this principle should be naturally satisfied in the context of holographic renormalization.

A closer look at the structure of the holographic renormalization equations (summarized in Appendix B) reveals a simple answer: In the procedure we followed in $3+1$ bulk dimensions, the potential terms in the counterterm at order four are generated by quadratic terms in the stress-energy tensor and field momenta at order two. These momenta arise from the functional differentiation of the counterterm at order two. Consider the counterterm appearing above at order two:

$$
S_{c t}^{(2)}=-\int_{\partial \mathcal{M}} d t d^{2} \mathbf{x} \sqrt{\gamma} N\left\{\frac{1}{32 \pi G_{4}}\left[\hat{R}+\frac{1}{2}\left(\frac{\nabla N}{N}\right)^{2}\right]-\frac{1}{4} \partial_{i} X^{a} \partial^{i} X^{a}\right\} .
$$

This Lagrangian is exactly the one used in the detailed balance condition in [1], in the case where $N$ does not depend upon spatial coordinates. ${ }^{9}$ Hence, the detailed balance relation, as reviewed in Section 2, is simply a consequence of the relationship between two counterterms implied by the holographic renormalization in asymptotically Lifshitz spacetime.

It should be noted that in the above procedure, the presence of the massive vector complicates the equations and make the detailed-balance-like relation between the two actions less transparent. But the logarithmic counterterm potential terms (with scaling dimension four) are nonetheless directly derivable from the counterterms with scaling dimension two.

In fact, an analogous result also holds in the relativistic case of holographic renormalization in $A d S_{5}$, where the second order counterterm is simply the Einstein-Hilbert action and the conformal anomaly is the action $S_{\text {conf }}$ of conformal gravity in $3+1$ dimensions: It turns out that $S_{\text {conf }}$ is obtained by squaring the functional derivative of the Einstein-Hilbert action. The reason behind this relationship is the same: $S_{\text {conf }}$ and the Einstein-Hilbert action appear as two counterterms, linked via the holographic renormalization procedure into a condition reminiscent of detailed balance.

A closer look also reveals that the holographic justification for the detailed balance condition being satisfied by the logarithmic conterterm quickly ceases to be valid with increasing spacetime dimension. However, this property does not disappear completely: Instead, the holographic renormalization machinery implies a more complex relation between the logarithmic counterterm and the variational derivatives of the entire hierarchy of the power-law counterterms.

\subsection{Analytic Continuation to the de Sitter-like Regime}

In relativistic $\mathrm{AdS} / \mathrm{CFT}$ correspondence, the Hamilton-Jacobi formulation of holographic renormalization - with the radial direction $r$ as the evolution parameter - can be easily

\footnotetext{
${ }^{9}$ Detailed balance in the nonprojectable theory has been discussed recently in [59].
} 
continued analytically to de Sitter space. Upon this continuation, the evolution parameter $r$ becomes the real time $\eta$, and the analytic continuation of the counterterms gives useful information about the wavefunction $\Psi$ of the Universe on superhorizon scales [27-29]. In particular, in the case of $A d S_{5}$ continued analytically to $d S_{5}$, the exponential of the logarithmic counterterm (known to take the form of the relativistic conformal gravity action $S_{\text {conf }}$ in $3+1$ dimensions) is related to the wavefunction via

$$
|\Psi|^{2}=e^{-S_{\text {conf }}}
$$

In this paper, we have analyzed holographic counterterms in the Lifshitz space background, and in the case of $z=2$ and $3+1$ bulk dimensions, we also found a logarithmic counterterm in the form of a $z=2$ multicritical conformal gravity action. It is natural to ask whether an analytic continuation exists, similar to the one studied in [27-29], so that the $z=2$ anisotropic conformal gravity action similarly produces the square of the wavefunction of the dual system. The answer appears to be yes, and the dual system is a gravity theory with an interesting kind of spatial anisotropy.

Reintroducing the length scale $L_{r}$ in the spacetime metric of the Lifshitz space at $z=2$,

$$
d s^{2}=L_{r}^{2}\left(-r^{4} d t^{2}+r^{2} d \mathbf{x}^{2}+\frac{d r^{2}}{r^{2}}\right)
$$

we can analytically continue our results by taking $r=i \eta$ and $L_{r}=-i L_{\eta}$ and relabeling $t=y$, which leads to the following spacetime:

$$
d s^{2}=L_{\eta}^{2}\left(\eta^{4} d y^{2}+\eta^{2} d \mathbf{x}^{2}-\frac{d \eta^{2}}{\eta^{2}}\right)
$$

This spacetime can be viewed as a spatially anisotropic, "multicritical" version of de Sitter space. We found the on-shell action for asymptotically Lifshitz space to be (with the cutoff at $\left.r=1 / \epsilon_{r}\right)$ :

$$
\begin{aligned}
S & =\frac{L_{r}^{2}}{16 \pi G_{4}} \int_{\partial \mathcal{M}_{1 / \epsilon_{r}}} d t d^{2} \mathbf{x} \sqrt{\gamma} N\left(\mathcal{L}^{(0)}+\mathcal{L}^{(2)}+\mathcal{L}^{(4)}-\widetilde{\mathcal{L}}^{(4)} \log \epsilon_{r}\right) \\
& =\frac{L_{r}^{2}}{16 \pi G_{4}} \int_{\partial \mathcal{M}_{1 / \epsilon_{r}}} d t d^{2} \mathbf{x} \sqrt{\gamma_{\text {fin }}} N_{\text {fin }}\left\{\frac{\mathcal{L}_{\text {fin }}^{(0)}}{\epsilon_{r}^{4}}+\frac{\mathcal{L}_{\text {fin }}^{(2)}}{\epsilon_{r}^{2}}+\mathcal{L}_{\text {fin }}^{(4)}-\widetilde{\mathcal{L}}_{\text {fin }}^{(4)} \log \epsilon_{r}\right\}
\end{aligned}
$$

where the quantities with fins are defined to be finite as $r \rightarrow \infty$ (that is, $\left.\mathcal{O}^{(\Delta)}=\mathcal{O}_{\text {fin }}^{(\Delta)} \epsilon_{r}^{\Delta}\right)$. The analytic continuation implies that the cutoff changes to $\epsilon_{r}=-i \epsilon_{\eta}$, where $\epsilon_{\eta}<0$. Note that all terms in the on-shell action remain real after the analytic continuation, except for the logarithm, which now has an imaginary part since $\log \epsilon_{r}=\log \left(-\epsilon_{\eta}\right)+i \pi / 2$. Thus, after this analytic continuation, the square of the ground-state wavefunction for the spatially anisotropic version of de Sitter space is given solely by the coefficient of the logarithmic counterterm,

$$
|\Psi|^{2}=\left|e^{i S}\right|^{2}=\exp \left\{-\frac{L_{\eta}^{2}}{16 G_{4}} \int_{\partial \mathcal{M}} d^{2} \mathbf{x} d y \sqrt{\gamma} N \widetilde{\mathcal{L}}^{(4)}\right\}
$$


In the case of the theory studied in Section 4.2 , we found that $\widetilde{\mathcal{L}}^{(4)}$ is the action of $z=2$ conformal Lifshitz gravity in detailed balance. It depends only on the $y$ derivatives but not the $\mathbf{x}$ derivatives of the metric. Thus, the ground-state wavefunction (4.51) represents a theory with spatial anisotropy, ultralocal along all but one spatial dimension, similar to the theory discussed in $[60,61]$.

In the theory with bulk scalars studied in Section $4.3, \widetilde{\mathcal{L}}^{(4)}$ was found to be the action of $z=2$ conformal Lifshitz gravity coupled to $z=2$ scalars, still satisfying the detailed balance condition. This action has a nontrivial potential term, of fourth order in the $\mathbf{x}$ derivatives of the scalars. Notably, the sign of this potential term, which we commented on at the end of Section 4.3, is such that the analytically continued $\widetilde{\mathcal{L}}^{(4)}$ appearing in (4.51) is positive definite.

\section{Conclusions}

The theory of gravity with anisotropic scaling introduced in $[1,2]$ has already been found to play a variety of roles in condensed matter. For example, linearized multicritical gravity with $z=2$ and $z=3$ emerges in the infrared regime of various bosonic lattice models, on a rigid lattice [62]: Gravitons with the nonrelativistic dispersion relation represent low-energy collective excitations of the lattice degrees of freedom. Dynamical gravity with anisotropic scaling also emerges naturally from fermionic condensed matter systems when the fundamental fermions are integrated out [63]. In the present paper, we have added another role to this list: Multicritical gravity naturally appears in the process of holographic renormalization of relativistic systems in spacetimes which are asymptotically anisotropic and describe holographic duals of nonrelativistic field theories. In the process, for the special case of bulk $2+1$ dimensions with $z=2$, we found that holographic renormalization imposes the condition of detailed balance on the action of $z=2$ conformal gravity coupled to matter, and gives a new rationale for this - otherwise somewhat obscure - condition.

Clearly, various interesting open questions remain. First of all, our analysis of holographic renormalization in the simplest anisotropic example, of $z=2$ in $3+1$ bulk dimensions, should generalize straightforwardly to higher integer values of $z$. Some calculations relevant for this task are reported in Appendix B. In particular, at $z=3$ in $4+1$ bulk dimensions, we expect the appearance of logarithmic counterterms taking the form of the action for $z=3$ multicritical conformal gravity in $3+1$ dimensions, introduced in [2]. Moreover, now that we have seen that the classical action of multicritical gravity appears from string-inspired holography, it would also be interesting to see whether the full dynamics of multicritical gravity can also be engineered from string theory, perhaps by taking judicious scaling limits of backgrounds without Lorentz invariance. Finally, it would also be natural to extend the study of nonrelativistic holography to the more general case, in which the bulk gravity itself exhibits spacetime anisotropies and multicriticality. ${ }^{10}$ Such constructions could extend the list of nonrelativistic field theories amenable to a holographic description to a broader class,

\footnotetext{
${ }^{10}$ Some early steps in that direction were suggested in $[1,2]$.
} 
in which those nonrelativistic theories that have a relativistic bulk dual may well be only a minority.

\section{Acknowledgments}

We wish to thank Yu Nakayama and Omid Saremi for useful discussions. This work has been supported by NSF Grant PHY-0855653, by DOE Grant DE-AC02-05CH11231, and by the Berkeley Center for Theoretical Physics.

\section{A. Notation and Conventions}

We use the following bulk metric:

$$
\begin{aligned}
d s^{2} & =G_{\mu \nu} d x^{\mu} d x^{\nu}=g_{\alpha \beta} d x^{\alpha} d x^{\beta}+\frac{d r^{2}}{r^{2}} \\
& =-N^{2} d t^{2}+\gamma_{i j}\left(d x^{i}+N^{i} d t\right)\left(d x^{j}+N^{j} d t\right)+\frac{d r^{2}}{r^{2}} .
\end{aligned}
$$

The boundary is at $r=\infty$. $D$ is the number of spatial dimensions on the boundary and so there are $D+2$ spacetime dimensions in the bulk and $d \equiv D+1$ spacetime dimensions on the boundary. For coordinate indices, $i, j$ are used for the $D$ spatial boundary indices $\left(x^{i}\right)$, whereas $\alpha, \beta$ are used for the $D+1$ spacetime boundary indices $\left(t, x^{i}\right)$ and $\mu, \nu$ are used for the $D+2$ bulk dimensions $\left(t, x^{i}, r\right)$. Note that in (A.1), the bulk diffeomorphisms have been gauge fixed by setting the the bulk shift vector $\mathcal{N}_{\alpha}$ (defined as $\mathcal{N}_{\alpha}=G_{r \alpha}$ ) to $\mathcal{N}_{\alpha}=0$, and the bulk lapse function (defined via $G_{r r}=\mathcal{N}^{2}+g^{\alpha \beta} \mathcal{N}_{\alpha} \mathcal{N}_{\beta}$ ) to $\mathcal{N}=1 / r$. This radial gauge is adopted throughout the paper. Moreover, in order to distinguish the lapse and shift variables in the bulk from those of the ADM decomposition on the boundary, we refer to the bulk variables $\mathcal{N}$ and $\mathcal{N}_{i}$ as the "radial lapse" and "radial shift."

It is often convenient to work in terms of vielbeins, which we define via

$$
\begin{aligned}
d s^{2} & =\eta_{M N} E_{\mu}^{M} E_{\nu}^{N} d x^{\mu} d x^{\nu}=\eta_{A B} e_{\alpha}^{A} e_{\beta}^{B} d x^{\alpha} d x^{\beta}+\frac{d r^{2}}{r^{2}} \\
& =-N^{2} d t^{2}+\delta_{I J} \hat{e}_{i}^{I} \hat{e}_{j}^{J}\left(d x^{i}+N^{i} d t\right)\left(d x^{j}+N^{j} d t\right)+\frac{d r^{2}}{r^{2}} .
\end{aligned}
$$

For the internal frame indices, $M, N=0,1, \ldots, D+1$ are used for the $D+2$ bulk dimensions, $A, B=0,1, \ldots, D$ are used for the $D+1$ spacetime boundary indices and $I, J=1, \ldots, D$ are used for the $D$ spatial boundary indices. The vielbeins allow coordinate indices to be changed to frame indices and vice versa, for example $F^{A B}=e_{\alpha}^{A} e_{\beta}^{B} F^{\alpha \beta}$. Also note that the vielbeins are related to the extrinsic curvature by $K_{\alpha \beta}=r\left(e_{\alpha}^{A} \partial_{r} e_{A \beta}+e_{\beta}^{A} \partial_{r} e_{A \alpha}\right) / 2$.

In order to distinguish the Riemann tensor and the extrinsic curvature tensor of the three different metrics $G_{\mu \nu}, g_{\alpha \beta}$ and $\gamma_{i j}$, we use the notation wherein $(D+2)$-dimensional quantities are written in curly letters (for example, $\mathcal{R}$ for the Ricci scalar), $(D+1)$-dimensional quantities are written in standard italics and $D$-dimensional quantities are written with hats. 


\section{A.1 The bulk action}

The bulk spacetime relativistic action is:

$$
\begin{gathered}
S_{\text {bulk }}=\frac{1}{16 \pi G_{D+2}} \int_{\mathcal{M}} d t d^{D} \mathbf{x} d r \sqrt{-G}\left(\mathcal{R}-2 \Lambda-\frac{1}{4} F_{\mu \nu} F^{\mu \nu}-\frac{1}{2} m^{2} \mathcal{A}_{\mu} \mathcal{A}^{\mu}\right) \\
+\frac{1}{8 \pi G_{D+2}} \int_{\partial \mathcal{M}} d t d^{D} \mathbf{x} \sqrt{-g} K .
\end{gathered}
$$

Note that in order for the Lifshitz spacetime (3.1) to be a classical solution, we set

$$
m^{2}=D z \quad \text { and } \quad \Lambda=-\frac{1}{2}\left(z^{2}+(D-1) z+D^{2}\right)
$$

The Lifshitz metric is sourced by a non-zero condensate of the vector field, and we denote by $\psi$ the deviation away from this non-zero background:

$$
\mathcal{A}_{A}=(\alpha+\psi) \delta_{A}^{0}
$$

with

$$
\alpha^{2}=\frac{2(z-1)}{z} .
$$

The leading order behavior of the vector at the boundary is $\psi \sim r^{-\Delta_{-}}$, where we use the notation of $[64,65]$ :

$$
\Delta_{-}=\frac{1}{2}\left(z+D-\beta_{z}\right)
$$

and

$$
\beta_{z}=\sqrt{(z+D)^{2}+8(z-1)(z-D)} .
$$

When the action (A.3) is evaluated as a function of the boundary fields we write it as:

$$
S=\frac{1}{16 \pi G_{D+2}} \int_{\partial \mathcal{M}} d t d^{D} \mathbf{x} \sqrt{\gamma} N \mathcal{L}
$$

\section{A.2 ADM decomposition in the metric formalism}

In our calculations, we decompose the metric $g_{\alpha \beta}$ on the $(D+1)$-dimensional boundary of spacetime into the ADM decomposition

$$
\begin{array}{rlrl}
g_{t t}=-N^{2}+N^{i} N_{i}, \quad g_{i j}=\gamma_{i j}, & g_{t i} & =N_{i}, \\
g^{t t}=-\frac{1}{N^{2}}, & g^{i j}=\gamma^{i j}-\frac{N^{i} N^{j}}{N^{2}}, & g^{t i} & =\frac{N^{i}}{N^{2}} .
\end{array}
$$

This metric leads to the following Christoffel symbols:

$$
\begin{aligned}
& \Gamma_{t t}^{t}=\frac{\partial_{t} N}{N}+\frac{N^{j} \nabla_{j} N}{N}+\frac{N^{i} N^{j} \hat{K}_{i j}}{N}, \\
& \Gamma_{t t}^{i}=\gamma^{i j} N \nabla_{j} N+N \gamma^{i j} \partial_{t}\left(\frac{N_{j}}{N}\right)-\frac{N^{i} N^{j} \nabla_{j} N}{N}-\gamma^{i j} N^{k} \nabla_{j} N_{k}-\frac{N^{i} N^{j} N^{k} \hat{K}_{j k}}{N},
\end{aligned}
$$




$$
\begin{aligned}
\Gamma_{t i}^{t} & =\frac{\nabla_{i} N}{N}+\frac{N^{j} \hat{K}_{i j}}{N} \\
\Gamma_{t i}^{j} & =N \gamma^{j k} \hat{K}_{i k}+N \nabla_{i}\left(\frac{N^{j}}{N}\right)-\frac{N^{j} N^{k} \hat{K}_{i k}}{N}, \\
\Gamma_{i j}^{t} & =\frac{\hat{K}_{i j}}{N} \\
\Gamma_{i j}^{k} & =\hat{\Gamma}_{i j}^{k}-\frac{\hat{K}_{i j} N^{k}}{N}
\end{aligned}
$$

where $\hat{K}_{i j}=\frac{1}{2 N}\left(\partial_{t} \gamma_{i j}-\nabla_{i} N_{j}-\nabla_{j} N_{i}\right)$ is the $D$-dimensional extrinsic curvature.

These result in the following $(D+1)$-dimensional Ricci scalar $R$ for the metric $g_{\alpha \beta}$ in terms of $\hat{R}$, the $D$-dimensional Ricci scalar for the metric $\gamma_{i j}$ :

$$
R=\hat{R}-\frac{2 \nabla^{2} N}{N}+\hat{K}_{i j} \hat{K}^{i j}-\hat{K}^{2}+\frac{\partial_{t} Z}{N \sqrt{\gamma}}+\frac{\nabla^{i} Y_{i}}{N}
$$

where:

$$
\begin{aligned}
Z & \equiv \gamma^{i j} \sqrt{\gamma} \nabla_{i}\left(\frac{N_{j}}{N}\right)+2 \hat{K} \sqrt{\gamma} \\
Y_{i} & \equiv-\partial_{t}\left(\frac{N_{i}}{N}\right)+\frac{N^{j} \nabla_{i} N_{j}}{N}+2 N^{j} \hat{K}_{i j}-3 N_{i} \hat{K}+\frac{N^{j} \nabla_{j} N_{i}}{N}-\frac{N_{i} \nabla_{j} N^{j}}{N} .
\end{aligned}
$$

\section{A.3 ADM decomposition in the vielbein formalism}

The vielbeins are defined by $g_{\alpha \beta}=e_{\alpha}^{A} e_{\beta}^{B} \eta_{A B}$ and $\gamma_{i j}=\hat{e}_{i}^{I} \hat{e}_{j}^{J} \delta_{I J}$. The $(D+1)$ dimensional boundary has vielbeins $e^{A}$ given by:

$$
e^{0}=N d t, \quad e^{I}=\hat{e}_{i}^{I}\left(N^{i} d t+d x^{i}\right)=N^{I} d t+\hat{e}^{I} .
$$

The Ricci rotation coefficients are defined by $d e^{C}=\Omega_{A B}{ }^{C} e^{A} \wedge e^{B}$,

$$
\begin{aligned}
& d e^{0}=\nabla_{i} N d x^{i} \wedge d t=\frac{\nabla_{I} N}{N} e^{I} \wedge e^{0}, \\
& d e^{I}=\left(\frac{\nabla_{J} N^{I}}{N}-\frac{\hat{e}_{J}^{j} \partial_{t} \hat{e}_{j}^{I}}{N}\right) e^{J} \wedge e^{0}+\hat{\Omega}_{J K}{ }^{I} e^{J} \wedge e^{K} .
\end{aligned}
$$

This means that:

$$
\begin{aligned}
\Omega_{0 I}{ }^{0} & =\frac{\nabla_{I} N}{2 N} \\
\Omega_{I J}{ }^{0} & =0 \\
\Omega_{0 J}{ }^{I} & =-\frac{\nabla_{J} N^{I}}{2 N}+\frac{\hat{e}_{J}^{j} \partial_{t} \hat{e}_{j}^{I}}{2 N}, \\
\Omega_{J K^{I}} & =\hat{\Omega}_{J K}^{I}, \\
\Omega_{0 I}{ }^{I} & =-\frac{\nabla_{I} N^{I}}{2 N}+\frac{\hat{e}_{I}^{j} \partial_{t} \hat{e}_{j}^{I}}{2 N}=-\frac{\nabla_{I} N^{I}}{2 N}+\frac{\gamma^{i j} \partial_{t} \gamma_{i j}}{4 N}=\frac{\hat{K}}{2} .
\end{aligned}
$$


Note that by definition $\Omega_{A B}{ }^{C}=-\Omega_{B A}{ }^{C}$. The covariant derivative is then given by:

$$
\nabla_{\alpha} V_{B}=\partial_{\alpha} V_{B}-\omega_{\alpha B}{ }^{C} V_{C}
$$

where $\omega_{A B C}=-\Omega_{A B C}+\Omega_{A C B}+\Omega_{B C A}$. Note that $\omega_{A B C}=-\omega_{A C B}$. Also $\omega_{[A B]}^{C}=-\Omega_{A B}{ }^{C}$ and $\omega_{C D}{ }^{C}=2 \Omega_{D C}{ }^{C}$.

\section{A.4 The massive vector}

We take the massive vector to be $\mathcal{A}_{A}=(\alpha+\psi) \delta_{A}^{0}$ where $\alpha^{2}=2(z-1) / z$. Also, the massive vector has a non-zero component in the $r$ direction, which the equation of motion for $\mathcal{A}_{r}$ gives as $\mathcal{A}_{r}=-\frac{\nabla^{\alpha} F_{r \alpha}}{m^{2}}=-\frac{\nabla^{A} \pi_{A}}{m^{2} r}$. Then:

$$
\mathcal{A}_{\alpha}=e_{\alpha}^{A} \mathcal{A}_{A}=e_{\alpha}^{0}(\alpha+\psi)=N(\alpha+\psi) \delta_{\alpha}^{t} .
$$

The only non-zero component of $F_{\alpha \beta}$ is

$$
F_{i t}=-F_{t i}=\partial_{i} \mathcal{A}_{t}=\alpha \nabla_{i} N+\nabla_{i}(N \psi) .
$$

The non-zero components of $F^{\alpha \beta}$ are

$$
F^{j t}=-F^{t j}=-\frac{\gamma^{i j} F_{i t}}{N^{2}}, \quad F^{j k}=\frac{\left(\gamma^{i j} N^{k}-\gamma^{i k} N^{j}\right) F_{i t}}{N^{2}} .
$$

Therefore we have that:

$$
\begin{aligned}
F_{A B} F^{A B}=F_{\alpha \beta} F^{\alpha \beta} & =-\frac{2\left(\alpha \nabla_{i} N+\nabla_{i}(N \psi)\right)\left(\alpha \nabla^{i} N+\nabla^{i}(N \psi)\right)}{N^{2}} \\
& =-2 \alpha^{2}\left(\frac{\nabla N}{N}\right)^{2}-4 \alpha \frac{\nabla_{i}(N \psi) \nabla^{i} N}{N^{2}}-\frac{2 \nabla_{i}(N \psi) \nabla^{i}(N \psi)}{N^{2}} .
\end{aligned}
$$

\section{A.5 Functional derivatives and the stress tensor}

We define the momenta corresponding to the metric $g_{\alpha \beta}$ and vector field $\mathcal{A}_{\alpha}$ by $\pi_{\alpha \beta}=$ $K_{\alpha \beta}-g_{\alpha \beta} K$ and $\pi_{\alpha}=r F_{r \alpha}$ respectively, ${ }^{11}$ where $K_{\alpha \beta}=r \partial_{r} g_{\alpha \beta} / 2$. As in the standard Hamilton-Jacobi theory, the momenta can also be obtained by functional differentiation of the on-shell action:

$$
\pi^{\alpha \beta}=-\frac{16 \pi G_{D+2}}{\sqrt{-g}} \frac{\delta S}{\delta g_{\alpha \beta}}, \quad \quad \pi^{\alpha}=-\frac{16 \pi G_{D+2}}{\sqrt{-g}} \frac{\delta S}{\delta \mathcal{A}_{\alpha}} .
$$

Equivalently, the variation of the on-shell action is:

$$
\begin{aligned}
\delta S & =-\frac{1}{16 \pi G_{D+2}} \int_{\partial \mathcal{M}} d^{d} x \sqrt{-g}\left[\pi^{\alpha \beta} \delta g_{\alpha \beta}+\pi^{\alpha} \delta \mathcal{A}_{\alpha}\right] \\
& =-\frac{1}{16 \pi G_{D+2}} \int_{\partial \mathcal{M}} d^{d} x \sqrt{-g}\left[\left(2 \pi^{\alpha}{ }_{\beta}+\pi^{\alpha} \mathcal{A}_{\beta}\right) e_{B}^{\beta} \delta e_{\alpha}^{B}+\pi^{A} \delta \mathcal{A}_{A}\right] .
\end{aligned}
$$

\footnotetext{
${ }^{11}$ This differs from the usual canonical momenta by a factor of $\sqrt{-g}\left(16 \pi G_{D+2}\right)^{-1}$ in order to simplify some of the subsequent equations.
} 
The boundary stress tensor $T^{\alpha}{ }_{B}$, however, is defined by functional differentiation of the onshell action with respect to the vielbeins $e_{\alpha}^{B}$, while holding the vector field with frame indices $\left(\mathcal{A}_{A}\right)$ fixed. Note that $\mathcal{A}_{0}=\alpha+\psi$ is the only non-zero component of $\mathcal{A}_{A}$. Therefore, the variation of the on-shell action can also be written as:

$$
\delta S=-\frac{1}{16 \pi G_{D+2}} \int d^{d} x \sqrt{\gamma} N\left[T^{\alpha}{ }_{B} \delta e_{\alpha}^{B}+\pi_{\psi} \delta \psi\right]
$$

where

$$
\begin{aligned}
T_{B}^{A} & =-\frac{16 \pi G_{D+2}}{\sqrt{\gamma} N} e_{\alpha}^{A} \frac{\delta S}{\delta e_{\alpha}^{B}}=-\frac{1}{\sqrt{\gamma} N} e_{\alpha}^{A} \frac{\delta}{\delta e_{\alpha}^{B}} \int d t d^{D} \mathbf{x} \sqrt{\gamma} N \mathcal{L} \\
\pi_{\psi} & =-\frac{16 \pi G_{D+2}}{\sqrt{\gamma} N} \frac{\delta S}{\delta \psi}=-\frac{1}{\sqrt{\gamma} N} \frac{\delta}{\delta \psi} \int d t d^{D} \mathbf{x} \sqrt{\gamma} N \mathcal{L}
\end{aligned}
$$

By comparing equations (A.28) and (A.29) we get the following relations:

$$
T_{\alpha B}=\left(2 \pi_{\alpha \beta}+\pi_{\alpha} \mathcal{A}_{\beta}\right) e_{B}^{\beta}, \quad \pi_{\psi}=\pi^{0} .
$$

Rearranging these expressions we have

$$
\pi_{A B}=\frac{1}{2}\left(T_{A B}-\pi_{A} \mathcal{A}_{B}\right), \quad \pi_{I} \mathcal{A}_{0}=T_{I 0}-T_{0 I} .
$$

Finally, by using the expressions for the vielbeins derived in Appendix A.3, we can write the stress tensor as:

$$
\begin{aligned}
T_{0}^{0} & =-\frac{16 \pi G_{D+2}}{\sqrt{\gamma}} \frac{\delta S}{\delta N}, \\
T^{0}{ }_{I} & =-\frac{16 \pi G_{D+2}}{\sqrt{\gamma}} \frac{\delta S}{\delta N^{I}}, \\
T^{I}{ }_{J} & =-16 \pi G_{D+2}\left(\frac{N^{I}}{\sqrt{\gamma} N} \frac{\delta S}{\delta N^{J}}+\frac{1}{\sqrt{\gamma} N} \hat{e}_{i}^{I} \frac{\delta S}{\delta \hat{e}_{i}^{J}}\right) \\
& =-16 \pi G_{D+2}\left(\frac{N^{I}}{\sqrt{\gamma} N} \frac{\delta S}{\delta N^{J}}+\frac{2}{\sqrt{\gamma} N} \hat{e}_{i}^{I} \hat{e}_{J j} \frac{\delta S}{\delta \gamma_{i j}}\right) .
\end{aligned}
$$

We will use these expressions to determine the stress tensor and vector momentum from the on-shell action

\section{A.6 Boundary source fields and asymptotic scaling}

The boundary conditions are specified by fixing the sources for the various field theory operators on the boundary. Our boundary conditions involve the following finite fixed sources as $r \rightarrow \infty$ (denoting each source with a bar):

$$
\bar{e}_{\alpha}^{0}=\frac{e_{\alpha}^{0}}{r^{z}}, \quad \bar{e}_{\alpha}^{I}=\frac{e_{\alpha}^{I}}{r}, \quad \bar{\psi}=\frac{\psi}{r^{-\Delta_{-}}} .
$$


In order to have a foliation on the boundary, it is necessary to set $\bar{e}_{i}^{0}$ (the source for the energy flux $\mathcal{E}^{i}$ ) equal to zero [42]. For all of this paper, we have set $\bar{e}_{i}^{0}=0$.

Note that $T^{\alpha}{ }_{A}$ is the vacuum expectation value of the operator sourced by $\bar{e}_{\alpha}^{A}$. In other words, $\bar{e}_{\alpha}^{0}$ is the source for the energy density $\mathcal{E}$ and the energy flux $\mathcal{E}^{i}$, whereas $\bar{e}_{\alpha}^{I}$ is the source for the momentum density $\mathcal{P}_{i}$ and the stress tensor $\Pi_{j}^{i} . \bar{\psi}$ is the source for $\mathcal{O}_{\psi}$, the operator dual to the massive vector $\psi$. The operator $\mathcal{O}_{\psi}$ is relevant for $z<D$ and irrelevant for $z>D$. Therefore, for $z>D$, we must take $\bar{\psi}=0$ in order to preserve the asymptotic boundary conditions above. In the case $z=D$, the operator is classically marginal, and there is some evidence suggesting that it becomes marginally relevant in the case of $D=2$ [66].

Note also that the scaling dimensions discussed here are the classical scaling dimensions, consistent with the fact that we perform our analysis near the ultraviolet fixed point with fixed $z$. In the bulk, this corresponds to the asymptotic analysis in the vicinity of the spacetime boundary at conformal infinity. Hence, in our analysis we systematically ignore most of the possible nontrivial infrared dynamics, such as the flow - generically expected of Lifshitz-type theories - towards lower values of $z$ under the influence of relevant operators.

The above scaling behavior allows us to determine the scaling behavior of other quantities near the boundary. Any boundary quantity can be written in terms of the source fields $\bar{e}_{\alpha}^{A}$ and $\bar{\psi}$ and then the scaling behavior can be read off from the resulting exponents of $r$. Consider a general object $\mathcal{O}$. When written in terms of the boundary source fields, we say that the term in $\mathcal{O}$ scaling as $r^{-\Delta}$ is of "order $\Delta$ " and denote it by $\mathcal{O}^{(\Delta)}$. For example, $e_{\alpha}^{0}$ has order $-z, e_{\alpha}^{I}$ has order -1 and $\psi$ has order $\Delta_{-}$. This means that $N$ has order $-z, N_{i}$ has order $-2, \gamma_{i j}$ has order -2 and $\gamma^{i j}$ has order 2 .

From equation (A.10), $R$ has components of order 2 and $2 z$ given by:

$$
\begin{aligned}
R^{(2)} & =\hat{R}-\frac{2 \nabla^{i} \nabla_{i} N}{N} \\
R^{(2 z)} & =\hat{K}_{i j} \hat{K}^{i j}-\hat{K}^{2}+\frac{\partial_{t} Z}{N \sqrt{\gamma}}+\frac{\nabla^{i} Y_{i}}{N} .
\end{aligned}
$$

From equation (A.25), $F_{A B} F^{A B}$ has components of order $2,2+\Delta_{-}$and $2+2 \Delta_{-}$given by:

$$
\begin{aligned}
\left(F_{A B} F^{A B}\right)^{(2)} & =-\frac{2 \alpha^{2} \nabla_{i} N \nabla^{i} N}{N^{2}}, \\
\left(F_{A B} F^{A B}\right)^{\left(2+\Delta_{-}\right)} & =-\frac{4 \alpha \nabla_{i}(N \psi) \nabla^{i} N}{N^{2}} \\
\left(F_{A B} F^{A B}\right)^{\left(2+2 \Delta_{-}\right)} & =-\frac{2 \nabla_{i}(N \psi) \nabla^{i}(N \psi)}{N^{2}} .
\end{aligned}
$$

Also, $\mathcal{A}_{A} \mathcal{A}^{A}=-(\alpha+\psi)^{2}$ has components of dimension $0, \Delta_{-}, 2 \Delta_{-}$:

$$
\begin{aligned}
\left(\mathcal{A}_{A} \mathcal{A}^{A}\right)^{(0)} & =-\alpha^{2}, \\
\left(\mathcal{A}_{A} \mathcal{A}^{A}\right)^{\left(\Delta_{-}\right)} & =-2 \alpha \psi, \\
\left(\mathcal{A}_{A} \mathcal{A}^{A}\right)^{\left(2 \Delta_{-}\right)} & =-\psi^{2} .
\end{aligned}
$$


Note also that equations (A.30) and A.31) imply that terms $\mathcal{L}^{(\Delta)}$ in the on-shell action determine $T_{0}^{0}{ }^{(\Delta)}, T_{I}^{0}{ }^{(\Delta+1-z)}, T_{0}^{I}{ }^{(\Delta+z-1)}, T_{J}^{I}{ }^{(\Delta)}$ and $\pi_{\psi}{ }^{\left(\Delta-\Delta_{-}\right)}$.

\section{B. Holographic Renormalization Equations}

The on-shell action is a function of the boundary fields and is written as

$$
S=\frac{1}{16 \pi G_{D+2}} \int d t d^{D} \mathbf{x} \sqrt{\gamma} N \mathcal{L}
$$

A convenient way of computing the divergent part of $\mathcal{L}$ is to organize the terms with respect to how they scale with $r$. More precisely, we define the dilatation operator by:

$$
\delta_{\mathcal{D}}=\int d t d^{D} \mathbf{x}\left(z e_{\mu}^{0} \frac{\delta}{\delta e_{\mu}^{0}}+e_{\mu}^{I} \frac{\delta}{\delta e_{\mu}^{I}}-\Delta_{-} \psi \frac{\delta}{\delta \psi}\right) .
$$

This operator asymptotically represents $r \frac{\partial}{\partial r}$.

$\mathcal{L}$ can then be decomposed into a sum of terms as follows:

$$
\mathcal{L}=\sum_{\Delta \geq 0} \mathcal{L}^{(\Delta)}+\widetilde{\mathcal{L}}^{(z+D)} \log r
$$

Note that we include a logarithmic term at order $z+D$ due to the possibility of a Weyl scaling anomaly. The individual terms of the expansion (B.3) satisfy

$$
\begin{gathered}
\delta_{\mathcal{D}} \mathcal{L}^{(\Delta)}=-\Delta \mathcal{L}^{(\Delta)} \quad \text { for } \quad \Delta \neq z+D, \\
\delta_{\mathcal{D}} \mathcal{L}^{(z+D)}=-(z+D) \mathcal{L}^{(z+D)}+\widetilde{\mathcal{L}}^{(z+D)}, \\
\delta_{\mathcal{D}} \widetilde{\mathcal{L}}^{(z+D)}=-(z+D) \widetilde{\mathcal{L}}^{(z+D)} .
\end{gathered}
$$

Applying $\delta_{\mathcal{D}}$ to the on-shell action (B.1) and using equations (A.30) and A.31) then yields:

$$
\left(z+D+\delta_{\mathcal{D}}\right) \mathcal{L}=-z T_{0}^{0}-T_{I}^{I}+\Delta_{-} \psi \pi_{\psi} .
$$

Expanding this at each order then results in:

$$
(z+D-\Delta) \mathcal{L}^{(\Delta)}=-z T_{0}^{0}{ }^{(\Delta)}-T_{I}^{I}{ }^{(\Delta)}+\Delta_{-} \psi \pi_{\psi}^{\left(\Delta-\Delta_{-}\right)}
$$

except for $\Delta=z+D$, when this becomes

$$
\widetilde{\mathcal{L}}^{(z+D)}=-z T_{0}^{0}{ }^{(z+D)}-T_{I}^{I}{ }^{(z+D)}+\Delta_{-} \psi \pi_{\psi}^{\left(z+D-\Delta_{-}\right)} .
$$

This allows us to solve for the anomaly. The above equations imply that the anomaly term can also be found by:

$$
\widetilde{\mathcal{L}}^{(\Delta)}=\lim _{\Delta \rightarrow z+D}\left((z+D-\Delta) \mathcal{L}^{(\Delta)}\right) .
$$


Note that the value of $\mathcal{L}^{(z+D)}$ cannot be found by this asymptotic analysis.

We now move on to finding an explicit expression for these divergent terms in the onshell action $\mathcal{L}^{(\Delta)}$. The variation of the bulk action (A.3) with respect to $\mathcal{N}$ produces the Hamiltonian constraint equation,

$$
K^{2}-K_{A B} K^{A B}-\frac{1}{2} \pi_{A} \pi^{A}-\frac{1}{2 m^{2}}\left(\nabla^{A} \pi_{A}\right)^{2}=R-2 \Lambda-\frac{1}{4} F_{A B} F^{A B}-\frac{1}{2} m^{2} \mathcal{A}_{A} \mathcal{A}^{A} .
$$

Expanding this equation in its dilatation eigenvalues (utilizing equations (A.33), (B.27), (B.29), (B.30) ) and then substituting it into equation (B.8) yields an expression for $\mathcal{L}^{(\Delta)}$ (see [42] for more details). Explicitly, the terms in the on-shell action are given for $\Delta \neq 0$, $\Delta_{-}$and $2 \Delta_{-}$by:

$$
(z+D-\Delta) \mathcal{L}^{(\Delta)}=\mathcal{Q}^{(\Delta)}+\mathcal{S}^{(\Delta)},
$$

where the quadratic term $\mathcal{Q}^{(\Delta)}$ is given by

$$
\begin{aligned}
& \mathcal{Q}^{(\Delta)}=\sum_{0<s<\Delta / 2 ; s \neq \Delta_{-}} {\left[2 K_{A B}^{(s)} \pi^{A B(\Delta-s)}+\pi_{A}^{(s)} \pi^{A(\Delta-s)}+\frac{1}{m^{2}}\left(\nabla_{A} \pi^{A}\right)^{(s)}\left(\nabla_{A} \pi^{A}\right)^{(\Delta-s)}\right] } \\
&+\left[K_{A B}^{\left(\Delta_{-}\right)} T^{A B\left(\Delta-\Delta_{-}\right)}+K_{00}^{\left(\Delta_{-}\right)} \pi^{0\left(\Delta-2 \Delta_{-}\right)} \psi+\pi_{I}^{\left(\Delta_{-}\right)} \pi^{I\left(\Delta-\Delta_{-}\right)}\right] \\
&+\left[K_{A B}^{(\Delta / 2)} \pi^{A B(\Delta / 2)}+\frac{1}{2} \pi_{A}^{(\Delta / 2)} \pi^{A(\Delta / 2)}+\frac{1}{2 m^{2}}\left(\nabla_{A} \pi^{A}\right)^{(\Delta / 2) 2}\right](\mathrm{B}
\end{aligned}
$$

and the source $\mathcal{S}$ is

$$
\mathcal{S}=R-2 \Lambda-\frac{1}{4} F_{A B} F^{A B}-\frac{1}{2} m^{2} \mathcal{A}_{A} \mathcal{A}^{A}
$$

We also have the following exceptions to the above formula:

$$
\begin{aligned}
(z+D) \mathcal{L}^{(0)} & =2 \mathcal{S}^{(0)} \\
\left(z+D-\Delta_{-}\right) \mathcal{L}^{\left(\Delta_{-}\right)} & =\left(\Delta_{-}-z\right) \psi \pi_{\psi}^{(0)}+\mathcal{S}^{\left(\Delta_{-}\right)} \\
\left(z+D-2 \Delta_{-}\right) \mathcal{L}^{\left(2 \Delta_{-}\right)} & =\left(\Delta_{-}-z\right) \psi \pi_{\psi}^{\left(\Delta_{-}\right)}+K_{A B}^{\left(\Delta_{-}\right)} \pi^{A B\left(\Delta_{-}\right)} \\
& +\frac{1}{2} \pi_{A}^{\left(\Delta_{-}\right)} \pi^{A\left(\Delta_{-}\right)}+\mathcal{S}^{\left(2 \Delta_{-}\right)} .
\end{aligned}
$$

$\mathcal{S}$ needs to be calculated at each order. The calculation in Appendix A.6 shows that $R$ has components of order 2 and $2 z, F_{A B} F^{A B}$ has components of order $2,2+\Delta_{-}, 2+2 \Delta_{-}$and $\mathcal{A}_{A} \mathcal{A}^{A}$ has components of order $0, \Delta_{-}, 2 \Delta_{-}$, resulting in:

$$
\begin{aligned}
\mathcal{S}^{(0)} & =-2 \Lambda+\frac{1}{2} m^{2} \alpha^{2}=(z+D)(z+D-1), \\
\mathcal{S}^{\left(\Delta_{-}\right)} & =m^{2} \alpha \psi=D z \alpha \psi \\
\mathcal{S}^{\left(2 \Delta_{-}\right)} & =\frac{1}{2} m^{2} \psi^{2}=\frac{D z}{2} \psi^{2}, \\
\mathcal{S}^{(2)} & =R^{(2)}-\frac{1}{4}\left(F_{A B} F^{A B}\right)^{(2)}=\hat{R}-2 \frac{\nabla^{2} N}{N}+\frac{\alpha^{2}}{2}\left(\frac{\nabla N}{N}\right)^{2},
\end{aligned}
$$




$$
\begin{aligned}
\mathcal{S}^{\left(2+\Delta_{-}\right)} & =-\frac{1}{4}\left(F_{A B} F^{A B}\right)^{\left(2+\Delta_{-}\right)}=\frac{\alpha \nabla_{i} N \nabla^{i}(N \psi)}{N^{2}} \\
\mathcal{S}^{\left(2+2 \Delta_{-}\right)} & =-\frac{1}{4}\left(F_{A B} F^{A B}\right)^{\left(2+2 \Delta_{-}\right)}=\frac{\nabla^{i}(N \psi) \nabla_{i}(N \psi)}{2 N^{2}} \\
\mathcal{S}^{(2 z)} & =R^{(2 z)}=\hat{K}_{i j} \hat{K}^{i j}-\hat{K}^{2}+\text { total derivatives. }
\end{aligned}
$$

We now proceed to use these formulae to calculate the divergent terms in the on-shell action at each order. Once these divergent terms have been calculated, counterterms must be added

to the action in order to subtract these divergences. With a boundary cutoff at $r=\frac{1}{\epsilon}$ ), the counterterms are

$$
S_{c t}=-\frac{1}{16 \pi G_{D+2}} \int d t d^{D} \mathbf{x} \sqrt{\gamma} N\left(\sum_{0 \leq \Delta<z+D} \mathcal{L}^{(\Delta)}-\widetilde{\mathcal{L}}^{(z+D)} \log \epsilon\right) .
$$

\section{B.1 Non-derivative counterterms}

At order 0, we have:

$$
\mathcal{L}^{(0)}=\frac{2 \mathcal{S}^{(0)}}{z+D}=2(z+D-1)
$$

This yields $T^{A}{ }_{B}{ }^{(0)}=-2(z+D-1) \delta^{A}{ }_{B}$.

To evaluate the order $\Delta_{-}$and $2 \Delta_{-}$counterterms we need some additional information. From the asymptotic expansions given in [42], it is clear that:

$$
K_{0}^{0}{ }^{(0)}=z, \quad K_{J}^{I}{ }^{(0)}=\delta^{I}{ }_{J}
$$

Also, the zero-component of the vector momentum is given by:

$$
\pi_{0}=r F_{r 0}=r \partial_{r} \mathcal{A}_{0}+\mathcal{A}_{0} K^{0}{ }_{0}-r \partial_{0} \mathcal{A}_{r} .
$$

This gives:

$$
\begin{aligned}
\pi_{0}^{(0)} & =\alpha K^{0}{ }_{0}{ }^{(0)}=\alpha z \\
\pi_{0}^{\left(\Delta_{-}\right)} & =r \partial_{r} \psi+\alpha K^{0}{ }_{0}^{\left(\Delta_{-}\right)}+\psi K^{0}{ }_{0}{ }^{(0)}=\alpha K^{0}{ }_{0}{ }^{\left(\Delta_{-}\right)}+\left(z-\Delta_{-}\right) \psi
\end{aligned}
$$

Note that $\pi_{\psi} \equiv \pi^{0}$.

Then:

$$
\begin{aligned}
\left(z+D-\Delta_{-}\right) \mathcal{L}^{\left(\Delta_{-}\right)} & =-\left(z-\Delta_{-}\right) \psi \pi_{\psi}^{(0)}+\mathcal{S}^{\left(\Delta_{-}\right)}=\left(z-\Delta_{-}\right) \psi \alpha z+D z \alpha \psi \\
\mathcal{L}^{\left(\Delta_{-}\right)} & =z \alpha \psi
\end{aligned}
$$

which yields $T^{A}{ }_{B}^{\left(\Delta_{-}\right)}=-z \alpha \psi \delta^{A}{ }_{B}$. 
Note that $\pi_{B}^{A}=\frac{1}{2}\left(T^{A}{ }_{B}-\pi^{A} \mathcal{A}_{B}\right)=K^{A}{ }_{B}-K \delta^{A}{ }_{B}$ and this means that:

$$
\begin{aligned}
\pi_{0}^{0}{ }^{\left(\Delta_{-}\right)} & =\frac{1}{2}\left(T_{0}^{0}{ }^{\left(\Delta_{-}\right)}-\alpha \pi_{\psi}^{\left(\Delta_{-}\right)}-\psi \pi_{\psi}^{(0)}\right)=-\frac{1}{2} \alpha \pi_{\psi}^{\left(\Delta_{-}\right)} \\
\pi_{J}^{I}{ }^{\left(\Delta_{-}\right)} & =\frac{1}{2} T^{I}{ }_{J}^{\left(\Delta_{-}\right)}=-\frac{z \alpha \psi}{2} \delta^{I}{ }_{J} \\
K^{\left(\Delta_{-}\right)} & =-\frac{\pi_{A}^{A}{ }^{\left(\Delta_{-}\right)}}{D}=\frac{\alpha \pi_{\psi}^{\left(\Delta_{-}\right)}}{2 D}+\frac{z \alpha \psi}{2} \\
K^{0}{ }_{0}^{\left(\Delta_{-}\right)} & =\pi_{0}^{0}{ }^{\left(\Delta_{-}\right)}+K^{\left(\Delta_{-}\right)}=-\frac{\alpha \pi_{\psi}^{\left(\Delta_{-}\right)}(D-1)}{2 D}+\frac{z \alpha \psi}{2} \\
K_{J}^{I}{ }^{\left(\Delta_{-}\right)} & =\pi_{J}^{I}{ }^{\left(\Delta_{-}\right)}+K^{\left(\Delta_{-}\right)} \delta^{I}{ }_{J}=\frac{\alpha \pi_{\psi}^{\left(\Delta_{-}\right)}}{2 D} \delta^{I}{ }_{J}
\end{aligned}
$$

Substituting this into the expression $\pi_{0}^{\left(\Delta_{-}\right)}=\alpha K_{0}^{0}{ }^{\left(\Delta_{-}\right)}+\left(z-\Delta_{-}\right) \psi$ derived above gives:

$$
\begin{aligned}
& \pi_{0}^{\left(\Delta_{-}\right)}=\alpha\left(-\frac{\alpha \pi_{\psi}^{\left(\Delta_{-}\right)}(D-1)}{2 D}+\frac{z \alpha \psi}{2}\right)+\left(z-\Delta_{-}\right) \psi \\
& \pi_{0}^{\left(\Delta_{-}\right)}=\frac{2 D\left(2 z-1-\Delta_{-}\right)}{2 D-\alpha^{2}(D-1)} \psi=\frac{D z\left(2 z-1-\Delta_{-}\right)}{z+D-1} \psi
\end{aligned}
$$

Therefore, using this result for $\pi_{0}^{\left(\Delta_{-}\right)}$:

$$
\begin{aligned}
K^{\left(\Delta_{-}\right)} & =-\frac{\alpha z\left(2 z-1-\Delta_{-}\right)}{2(z+D-1)} \psi+\frac{z \alpha \psi}{2}=-\frac{\alpha z\left(z-D-\Delta_{-}\right)}{2(z+D-1)} \psi \\
K_{0}^{0}{ }^{\left(\Delta_{-}\right)} & =\frac{\alpha z(D-1)\left(2 z-1-\Delta_{-}\right)}{2(z+D-1)} \psi+\frac{z \alpha \psi}{2}=\frac{\alpha z\left((2 D-1) z-(D-1) \Delta_{-}\right)}{2(z+D-1)} \psi( \\
K_{J}^{I}{ }^{\left(\Delta_{-}\right)} & =-\frac{\alpha z\left(2 z-1-\Delta_{-}\right)}{2(z+D-1)} \psi \delta_{J}^{I}
\end{aligned}
$$

Then:

$$
\begin{aligned}
\left(z+D-2 \Delta_{-}\right) \mathcal{L}^{\left(2 \Delta_{-}\right)}= & -\left(z-\Delta_{-}\right) \psi \pi_{\psi}^{\left(\Delta_{-}\right)}+K_{A B}^{\left(\Delta_{-}\right)} \pi^{A B\left(\Delta_{-}\right)}+\frac{1}{2} \pi_{A}^{\left(\Delta_{-}\right)} \pi^{A\left(\Delta_{-}\right)}+\mathcal{S}^{\left(2 \Delta_{-}\right)} \\
= & -\left(z-\Delta_{-}\right) \psi \pi_{\psi}^{\left(\Delta_{-}\right)}+\left(-\frac{\alpha \pi_{\psi}^{\left(\Delta_{-}\right)}(D-1)}{2 D}+\frac{z \alpha \psi}{2}\right)\left(-\frac{1}{2} \alpha \pi_{\psi}^{\left(\Delta_{-}\right)}\right) \\
& +\left(\frac{\alpha \pi_{\psi}^{\left(\Delta_{-}\right)}}{2}\right)\left(-\frac{z \alpha \psi}{2}\right)+\frac{1}{2}\left(\pi_{\psi}^{\left(\Delta_{-}\right)}\right)^{2}-\frac{D z}{2} \psi^{2} \\
= & \frac{D z \psi^{2}\left(4 z^{2}-4 z-4 z \Delta_{-}+1+2 \Delta_{-}+\Delta_{-}^{2}+z+D-1\right)}{2(z+D-1)} \\
= & \frac{D z \psi^{2}\left(z+D-2 \Delta_{-}\right)\left(2 z-1-\Delta_{-}\right)}{2(z+D-1)} \\
\mathcal{L}^{\left(2 \Delta_{-}\right)}= & \frac{D z \psi^{2}\left(2 z-1-\Delta_{-}\right)}{2(z+D-1)}
\end{aligned}
$$


where $\Delta_{-}=\frac{1}{2}\left(z+D-\beta_{z}\right)$ and $\beta_{z}=\sqrt{(z+D)^{2}+8(z-1)(z-D)}$ has been used.

This result yields $T_{B}^{A}{ }^{\left(2 \Delta_{-}\right)}=-\frac{D z \psi^{2}\left(2 z-1-\Delta_{-}\right)}{2(z+D-1)} \delta^{A}{ }_{B}$. Next we can calculate:

$$
\begin{aligned}
\left(z+D-3 \Delta_{-}\right) \mathcal{L}^{\left(3 \Delta_{-}\right)}= & K_{A B}^{\left(\Delta_{-}\right)} T^{A B\left(2 \Delta_{-}\right)}+K_{00}^{\left(\Delta_{-}\right)} \pi^{0(0)} \psi \\
= & \frac{D \alpha z^{2}\left(2 z-1-\Delta_{-}\right)\left(z-D-\Delta_{-}\right)}{4(z+D-1)^{2}} \psi^{3} \\
& +\frac{D \alpha z^{2}\left((2 D-1) z-(D-1) \Delta_{-}\right)\left(2 z-1-\Delta_{-}\right)}{2(z+D-1)^{2}} \psi^{3} \\
= & \frac{D \alpha z^{2}\left(2 z-1-\Delta_{-}\right)\left(-D+(4 D-1) z-(2 D-1) \Delta_{-}\right)}{4(z+D-1)^{2}} \psi^{3}
\end{aligned}
$$

which yields $\pi_{\psi}^{\left(2 \Delta_{-}\right)}=-\frac{3 D \alpha z^{2}\left(2 z-1-\Delta_{-}\right)\left(-D+(4 D-1) z-(2 D-1) \Delta_{-}\right)}{4\left(z+D-3 \Delta_{-}\right)(z+D-1)^{2}} \psi^{2}$.

This allows us to calculate $K_{A B}^{\left(2 \Delta_{-}\right)}$:

$$
\begin{aligned}
\pi_{0}^{0}{ }^{\left(2 \Delta_{-}\right)} & =\frac{1}{2}\left(T_{0}^{0}{ }^{\left(2 \Delta_{-}\right)}-\alpha \pi_{\psi}^{\left(2 \Delta_{-}\right)}-\psi \pi_{\psi}^{\left(\Delta_{-}\right)}\right)=-\frac{1}{2} \alpha \pi_{\psi}^{\left(2 \Delta_{-}\right)}-\frac{1}{4} \psi \pi_{\psi}^{\left(\Delta_{-}\right)} \\
\pi_{J}^{I}{ }^{\left(2 \Delta_{-}\right)} & =\frac{1}{2} T_{J}^{I_{J}{ }^{\left(2 \Delta_{-}\right)}}=\frac{1}{4} \psi \pi_{\psi}^{\left(\Delta_{-}\right)} \delta^{I}{ }_{J} \\
K^{\left(2 \Delta_{-}\right)} & =-\frac{\pi_{A}^{A}{ }^{\left(\Delta_{-}\right)}}{D}=\frac{1}{2 D} \alpha \pi_{\psi}^{\left(2 \Delta_{-}\right)}-\frac{(D-1)}{4 D} \psi \pi_{\psi}^{\left(\Delta_{-}\right)} \\
K_{0}^{0}{ }^{\left(2 \Delta_{-}\right)} & =\pi_{0}^{0}{ }^{\left(2 \Delta_{-}\right)}+K^{\left(2 \Delta_{-}\right)}=-\frac{\alpha \pi_{\psi}^{\left(2 \Delta_{-}\right)}(D-1)}{2 D}-\frac{(2 D-1)}{4 D} \psi \pi_{\psi}^{\left(\Delta_{-}\right)} \\
K_{J}^{I}{ }^{\left(2 \Delta_{-}\right)} & =\pi_{J}^{I^{\left(2 \Delta_{-}\right)}}+K^{\left(2 \Delta_{-}\right)} \delta_{J}^{I}=\left(\frac{1}{2 D} \alpha \pi_{\psi}^{\left(2 \Delta_{-}\right)}+\frac{1}{4 D} \psi \pi_{\psi}^{\left(\Delta_{-}\right)}\right) \delta_{J}^{I}
\end{aligned}
$$

Higher order non-derivative terms can be calculated in a similar manner.

\section{B.2 Two-derivative counterterms with $\psi=0$}

Up to total derivatives, the divergent term in the on-shell action of order 2 is:

$$
(z+D-2) \mathcal{L}^{(2)}=\mathcal{S}^{(2)}=\hat{R}-\frac{2 \nabla^{i} \nabla_{i} N}{N}+\frac{\alpha^{2} \nabla^{i} N \nabla_{i} N}{2 N^{2}}=\hat{R}+\frac{\alpha^{2} \nabla^{i} N \nabla_{i} N}{2 N^{2}}
$$

This gives the following contribution to the stress tensor (see Appendix A.5):

$$
\begin{aligned}
(z+D-2) T_{00}^{(2)} & =\hat{R}-\frac{\alpha^{2} \nabla^{i} \nabla_{i} N}{N}+\frac{\alpha^{2} \nabla^{i} N \nabla_{i} N}{2 N^{2}}, \\
(z+D-2) T_{0 I}^{(3-z)}= & 0, \\
(z+D-2) T_{I J}^{(2)}= & 2 \hat{R}_{I J}-\frac{2 \nabla_{I} \nabla_{J} N}{N}+\frac{\alpha^{2} \nabla_{I} N \nabla_{J} N}{N^{2}}, \\
& +\delta_{I J}\left(-\hat{R}+\frac{2 \nabla^{i} \nabla_{i} N}{N}-\frac{\alpha^{2} \nabla^{i} N \nabla_{i} N}{2 N^{2}}\right), \\
(z+D-2) T_{I}^{I^{(2)}}= & -(D-2) \hat{R}+\frac{2(D-1) \nabla^{i} \nabla_{i} N}{N}-\frac{\alpha^{2}(D-2) \nabla^{i} N \nabla_{i} N}{2 N^{2}} .
\end{aligned}
$$


At order $2 z$ there is a contribution from the quadratic term $\frac{1}{2 m^{2}}\left(\nabla_{A} \pi^{A}\right)^{(z) 2}$. Note that:

$$
\begin{gathered}
\left(\nabla_{A} \pi^{A}\right)^{(z)}=\left(\partial_{A} \pi^{A}-\omega_{A}{ }^{A B} \pi_{B}\right)^{(z)}=\left(\partial_{A} \pi^{A}-2 \Omega_{A}{ }^{B A} \pi_{B}\right)^{(z)} \\
=\left(\partial_{0}\left(\pi^{0(0)}\right)-2 \Omega_{I}{ }^{0 I} \pi_{0}^{(0)}\right)=\left(\partial_{0}(-z \alpha)-2 \Omega_{I}{ }^{0 I} z \alpha\right) \\
=-\alpha z \hat{K}
\end{gathered}
$$

where expressions from Appendix A.3 have been used. Therefore, up to total derivatives:

$$
\begin{aligned}
(z+D-2 z) \mathcal{L}^{(2 z)} & =\mathcal{S}^{(2 z)}+\frac{1}{2 m^{2}}\left(\nabla_{A} \pi^{A}\right)^{(z) 2} \\
& =\hat{K}_{i j} \hat{K}^{i j}-\hat{K}^{2}+\frac{1}{2 m^{2}}(-\alpha z \hat{K})^{2} \\
& =\hat{K}_{i j} \hat{K}^{i j}-\frac{(1+D-z)}{D} \hat{K}^{2}
\end{aligned}
$$

\section{B.3 Two-derivative counterterms involving $\psi$}

We can also calculate various divergent terms involving $\psi$, for example:

$$
\begin{gathered}
\left(z+D-2-\Delta_{-}\right) \mathcal{L}^{\left(2+\Delta_{-}\right)}=K_{A B}^{\left(\Delta_{-}\right)} T^{A B(2)}+\frac{\alpha \nabla_{i} N \nabla^{i}(N \psi)}{N^{2}} \\
=-\frac{\alpha z \psi}{2(z+D-1)}\left[\left((2 D-1) z-(D-1) \Delta_{-}\right) T^{00(2)}+\left(2 z-1-\Delta_{-}\right) T^{I}{ }_{I}^{(2)}\right] \\
-\frac{\alpha \nabla^{i} \nabla_{i} N \psi}{N}+\frac{\alpha \nabla_{i} N \nabla^{i} N \psi}{N^{2}} \\
=-\frac{\alpha z \psi}{2(z+D-1)(z+D-2)}\left[\left((2 D-1) z-(D-1) \Delta_{-}\right)\left(\hat{R}-\frac{\alpha^{2} \nabla^{i} \nabla_{i} N}{N}+\frac{\alpha^{2} \nabla^{i} N \nabla_{i} N}{2 N^{2}}\right)\right. \\
\left.+\left(2 z-1-\Delta_{-}\right)\left(-(D-2) \hat{R}+\frac{2(D-1) \nabla^{i} \nabla_{i} N}{N}-\frac{\alpha^{2}(D-2) \nabla^{i} N \nabla_{i} N}{2 N^{2}}\right)\right] \\
\quad-\frac{\alpha \nabla^{i} \nabla_{i} N \psi}{N}+\frac{\alpha \nabla_{i} N \nabla^{i} N \psi}{N^{2}} .
\end{gathered}
$$

Or, by defining some constants:

$$
\mathcal{L}^{\left(2+\Delta_{-}\right)}=-\psi\left(c_{1} \hat{R}+c_{2} \frac{\nabla^{i} \nabla_{i} N}{N}+c_{3} \frac{\nabla_{i} N \nabla^{i} N}{N^{2}}\right)
$$

where:

$$
\begin{aligned}
c_{1} & =\frac{\alpha z\left(-2+D-\Delta_{-}+3 z\right)}{2(D-2+z)(D-1+z)\left(z+D-2-\Delta_{-}\right)} \\
c_{2} & =\frac{\alpha\left(4+2 D^{2}-\left(4+\left(\alpha^{2}-2\right) \Delta_{-}\right) z+\left(\alpha^{2}-2\right) z^{2}+D\left(\left(2+\left(\alpha^{2}-2\right) \Delta_{-}\right) z-2\left(\alpha^{2}-2\right) z^{2}-6\right)\right)}{2(D-2+z)(D-1+z)\left(z+D-2-\Delta_{-}\right)} \\
c_{3} & =\frac{\alpha\left(-8-4 D^{2}-\left(-12+\alpha^{2}\left(2+\Delta_{-}\right)\right) z+\left(3 \alpha^{2}-4\right) z^{2}+D\left(12+\left(\alpha^{2}-8\right) z\right)\right)}{4(D-2+z)(-1+D+z)\left(-2+D-\Delta_{-}+z\right)}
\end{aligned}
$$

(Note that for $z=D=2$ we have $c_{1}=\frac{1}{2}, c_{2}=\frac{1}{2}$ and $c_{3}=-\frac{1}{4}$.)

This results in:

$$
\pi_{\psi}^{(2)}=c_{1} \hat{R}+c_{2} \frac{\nabla^{i} \nabla_{i} N}{N}+c_{3} \frac{\nabla_{i} N \nabla^{i} N}{N^{2}}
$$


and also:

$$
\begin{aligned}
T_{00}^{\left(2+\Delta_{-}\right)}= & -c_{1} \psi \hat{R}-c_{2} \nabla^{i} \nabla_{i} \psi-c_{3} \frac{\nabla_{i} N \nabla^{i} N \psi}{N^{2}}+2 c_{3} \frac{\nabla_{i} \nabla^{i} N \psi}{N}+2 c_{3} \frac{\nabla^{i} N \nabla_{i} \psi}{N} \\
T_{0 I}^{\left(3-z+\Delta_{-}\right)}= & 0 \\
T_{I J}^{\left(2+\Delta_{-}\right)}= & \delta_{I J}\left(c_{1} \psi \hat{R}-c_{2} \frac{\nabla_{i} N \nabla^{i} \psi}{N}+c_{3} \frac{\nabla_{i} N \nabla^{i} N \psi}{N^{2}}\right) \\
& -2 c_{1} \psi \hat{R}_{I J}+c_{2} \frac{\nabla_{I} N \nabla_{J} \psi}{N}+c_{2} \frac{\nabla_{J} N \nabla_{I} \psi}{N}-2 c_{3} \frac{\nabla_{I} N \nabla_{J} N \psi}{N^{2}} \\
& -2 \delta_{I J} c_{1} \frac{\nabla^{i} \nabla_{i}(N \psi)}{N}+2 c_{1} \frac{\nabla_{I} \nabla_{J}(N \psi)}{N} \\
T_{I}^{I}{ }^{\left(2+\Delta_{-}\right)}= & (D-2)\left(c_{1} \psi \hat{R}-c_{2} \frac{\nabla_{i} N \nabla^{i} \psi}{N}+c_{3} \frac{\nabla_{i} N \nabla^{i} N \psi}{N^{2}}\right)-2 c_{1} \frac{\nabla^{i} \nabla_{i}(N \psi)}{N}
\end{aligned}
$$

There are many more two-derivative terms involving $\psi$. For example:

$$
\begin{aligned}
\left(z+D-2-2 \Delta_{-}\right) \mathcal{L}^{\left(2+2 \Delta_{-}\right)} & =2 K_{A B}^{\left(2 \Delta_{-}\right)} \pi^{A B(2)}+\pi_{A}^{\left(2 \Delta_{-}\right)} \pi^{A(2)} \\
& +K_{A B}^{\left(\Delta_{-}\right)} T^{A B\left(2+\Delta_{-}\right)}+K_{00}^{\left(\Delta_{-}\right)} \pi^{0(2)} \psi+\mathcal{S}^{\left(2+2 \Delta_{-}\right)}
\end{aligned}
$$

This has been calculated explicitly in the case $D=z=2$ :

$$
\mathcal{L}^{\left(2+2 \Delta_{-}\right)}=\psi^{2}\left(\frac{\nabla^{i} \nabla_{i} N}{8 N}-\frac{\nabla^{i} N \nabla_{i} N}{2 N^{2}}\right)+\frac{3}{4} \psi \nabla^{i} \nabla_{i} \psi
$$

\section{B.4 Four-derivative counterterms with $\psi=0$}

At fourth order we have:

$$
\begin{aligned}
& (z+D-4) \mathcal{L}^{(4)}=K_{A B}^{(2)} \pi^{A B(2)}+\frac{1}{2} \pi_{A}^{(2)} \pi^{A(2)} \\
& =\frac{1}{a_{0}}\left[a_{1}\left(\frac{\nabla_{i} N \nabla^{i} N}{N^{2}}\right)^{2}+a_{2} \frac{\nabla_{i} N \nabla^{i} N}{N^{2}} \hat{R}+a_{3} \frac{\nabla^{i} \nabla_{i} N}{N} \frac{\nabla_{j} N \nabla^{j} N}{N^{2}}\right. \\
& \left.\quad+a_{4} \frac{\nabla^{i} N \nabla^{j} N}{N^{2}} \hat{R}_{i j}+a_{5} \frac{\nabla^{i} \nabla_{i} N}{N} \hat{R}+a_{6}\left(\frac{\nabla^{i} \nabla_{i} N}{N}\right)^{2}+a_{7} \hat{R}_{i j}^{2}+a_{8} \hat{R}^{2}\right]
\end{aligned}
$$

where:

$$
\begin{aligned}
a_{0}= & -2 D z^{2}(-2+D+z)^{2}(-1+D+z)(-4+\beta+D+z)^{2} \\
a_{1}= & 32(z-1)^{3}+D^{4}(-11+z(6+z)) \\
& +D^{3}\left(52-3 \beta_{z}-z\left(77+2 \beta_{z}-\left(34+\beta_{z}\right) z+z^{2}\right)\right) \\
& +D^{2}\left(16\left(-8+\beta_{z}\right)+z\left(2\left(116+\beta_{z}\right)+z\left(-145-8 \beta_{z}+2\left(9+\beta_{z}\right) z+3 z^{2}\right)\right)\right) \\
& +D(z-1)\left(16\left(-8+\beta_{z}\right)+z\left(184+z\left(-68-5 \beta_{z}+z\left(-13+\beta_{z}+5 z\right)\right)\right)\right) \\
a_{2}= & 2 z(z-1)\left(D^{4}+D^{3}\left(\beta_{z}-z\right)+16(z-1) z+D^{2}\left(8-4 \beta_{z}+z\left(-16+2 \beta_{z}+3 z\right)\right)\right. \\
& \left.+D z\left(-4\left(-8+\beta_{z}\right)+z\left(-24+\beta_{z}+5 z\right)\right)\right) \\
a_{3}= & -2 z\left(D^{4}(z-4)+32(z-1)^{2}+D^{3}\left(-2\left(7+2 \beta_{z}\right)+\left(21+\beta_{z}-z\right) z\right)\right. \\
& +D^{2}\left(32+18 \beta_{z}+z\left(-60-11 \beta_{z}+z\left(10+2 \beta_{z}+3 z\right)\right)\right)
\end{aligned}
$$




$$
\begin{aligned}
& \left.+D(z-1)\left(8\left(8+\beta_{z}\right)+z\left(-40-6 \beta_{z}+z\left(-18+\beta_{z}+5 z\right)\right)\right)\right) \\
a_{4}= & -4 z D(z-2)(D-1+z)\left(8+D^{2}-8 z-2 D z+5 z^{2}+\beta_{z}(-4+D+z)\right) \\
a_{5}= & -4 z^{2}\left(D^{3}+D^{2}\left(\beta_{z}-2 z\right)+8(z-1) z+D\left(8+\beta_{z}(z-4)-3 z^{2}\right)\right) \\
a_{6}= & -4 z(D-1)\left(D^{3}+D^{2}\left(\beta_{z}-2 z\right)+8(z-1) z+D\left(8+\beta_{z}(z-4)-3 z^{2}\right)\right) \\
a_{7}= & -4 z^{2} D(D-1+z)\left(8+D^{2}-8 z-2 D z+5 z^{2}+\beta_{z}(D-4+z)\right) \\
a_{8}= & z^{2}\left(D^{4}+D^{3}\left(\beta_{z}-z\right)+8(z-1) z^{2}+D^{2}\left(8-4 \beta_{z}+z\left(-8+2 \beta_{z}+3 z\right)\right)\right. \\
& \left.+D z\left(8-4 \beta_{z}+z\left(-8+\beta_{z}+5 z\right)\right)\right)
\end{aligned}
$$

In the above expression we have used the following identities for terms in the action (up to total derivatives):

$$
\begin{aligned}
\frac{\nabla_{i} N \nabla_{j} N \nabla^{i} \nabla^{j} N}{N^{3}} & \sim\left(\frac{\nabla_{i} N \nabla^{i} N}{N^{2}}\right)^{2}-\frac{\nabla_{i} N \nabla^{i} N \nabla^{j} \nabla_{j} N}{2 N^{3}} \\
\frac{\nabla_{i} \nabla_{j} N \nabla^{i} \nabla^{j} N}{N^{2}} & \sim\left(\frac{\nabla_{i} N \nabla^{i} N}{N^{2}}\right)^{2}-\frac{3 \nabla_{i} N \nabla^{i} N \nabla^{j} \nabla_{j} N}{2 N^{3}}+\left(\frac{\nabla^{i} \nabla_{i} N}{N}\right)^{2}-\frac{\nabla^{i} N \nabla^{j} N R_{i j}}{N^{2}} \\
\frac{\nabla^{i} \nabla^{j} N R_{i j}}{N} & \sim \frac{\nabla^{i} \nabla_{i} N R}{2 N}
\end{aligned}
$$

For $D=2$ we have further simplifications because $R_{i j}=\frac{R}{2} \delta_{i j}$ and so:

$$
\begin{gathered}
(z-2) \mathcal{L}^{(4)}=\frac{(z-2)}{b_{0}}\left[b_{1}\left(\frac{\nabla_{i} N \nabla^{i} N}{N^{2}}\right)^{2}+b_{2} \frac{\nabla_{i} N \nabla^{i} N}{N^{2}} \hat{R}+b_{3} \frac{\nabla^{i} \nabla_{i} N}{N} \frac{\nabla_{j} N \nabla^{j} N}{N^{2}}\right. \\
\left.+b_{4} \frac{\nabla^{i} \nabla_{i} N}{N} \hat{R}+b_{5}\left(\frac{\nabla^{i} \nabla_{i} N}{N}\right)^{2}+b_{6} \hat{R}^{2}\right]
\end{gathered}
$$

where:

$$
\begin{aligned}
& b_{0}=-2 z^{4}(z+1)\left(z-2+\beta_{z}\right)^{2} \\
& b_{1}=12+36 z-11 z^{2}-2 z^{3}+5 z^{4}+\beta_{z}\left(-2-7 z+z^{3}\right) \\
& b_{2}=4 z^{2}\left(z-6+\beta_{z}\right) \\
& b_{3}=-2 z\left(36-4 z-7 z^{2}+5 z^{3}+\beta_{z}\left(z^{2}-z-6\right)\right) \\
& b_{4}=-4 z^{2}\left(z-6+\beta_{z}\right) \\
& b_{5}=-4 z\left(z-6+\beta_{z}\right) \\
& b_{6}=-z^{3}\left(z-6+\beta_{z}\right)
\end{aligned}
$$

Note that in the important case where $z \rightarrow 2$ (and still $D=2$ ):

$$
(z-2) \mathcal{L}^{(4)}=\frac{(2-z)}{64}\left[3\left(\frac{\nabla_{i} N \nabla^{i} N}{N^{2}}\right)^{2}-4 \frac{\nabla^{i} \nabla_{i} N}{N} \frac{\nabla_{j} N \nabla^{j} N}{N^{2}}\right]
$$


A useful check is for $z=1$, which is the usual relativistic AdS case. The standard known result [47] is that the 4th order term involving only spatial derivative is (up to total derivatives):

$$
\begin{aligned}
\mathcal{L}^{(4)}= & {\left[\frac{1}{(D-3)(D-1)^{2}}\left(R_{\alpha \beta} R^{\alpha \beta}-\frac{D+1}{4 D} R^{2}\right)\right]^{(4)} } \\
= & \frac{1}{(D-3)(D-1)^{2}}\left[\left(\frac{\nabla^{i} \nabla_{i} N}{N}\right)^{2}+\left(\hat{R}_{i j}-\frac{\nabla_{i} \nabla_{j} N}{N}\right)\left(\hat{R}^{i j}-\frac{\nabla^{i} \nabla^{j} N}{N}\right)\right. \\
& \left.\quad-\frac{D+1}{4 D}\left(\hat{R}-\frac{2 \nabla^{i} \nabla_{i} N}{N}\right)^{2}\right] \\
= & -\frac{1}{(D-3)(D-1)^{2}}\left[-\left(\frac{\nabla_{i} N \nabla^{i} N}{N^{2}}\right)^{2}+\frac{3 \nabla_{i} N \nabla^{i} N \nabla^{j} \nabla_{j} N}{2 N^{3}}+\frac{\nabla^{i} N \nabla^{j} N \hat{R}_{i j}}{N^{2}}\right. \\
& \left.-\frac{1}{D} \frac{\nabla_{i} \nabla^{i} N}{N} \hat{R}-\frac{D-1}{D}\left(\frac{\nabla^{i} \nabla_{i} N}{N}\right)^{2}-\hat{R}_{i j} \hat{R}^{i j}+\frac{D+1}{4 D} \hat{R}^{2}\right]
\end{aligned}
$$

This agrees exactly with the general result above. Of course, for $z=1$ there will also be contributing terms at this order which come from the $4 z$ and $2+2 z$ order terms (these will involve time derivatives).

An easily computable case is $D=1$ (for which $\hat{R}=0$ ). The above expressions yield $(z-3) \mathcal{L}^{(4)}=\frac{(z-3) \nabla_{i} N \nabla^{i} N}{12 z^{3} N^{2}}$. For $z=3$, which is when this would possibly generate a scaling anomaly, this expression vanishes.

\section{B.5 Four-derivative counterterms involving $\psi$}

There are many possible four-derivative counterterms involving $\psi$, for example:

$$
\left(z+D-4-\Delta_{-}\right) \mathcal{L}^{\left(4+\Delta_{-}\right)}=2 K_{A B}^{(2)} \pi^{A B\left(2+\Delta_{-}\right)}+\pi_{A}^{(2)} \pi^{A\left(2+\Delta_{-}\right)}+K_{A B}^{\left(\Delta_{-}\right)} T^{A B(4)} .
$$

The right hand-side has been explicitly calculated and found to be zero in the case where $z=2$ and $D=2$.

\section{Anisotropic Weyl Anomaly in $2+1$ Dimensions}

Just as in the relativistic case, a theory which has the classical symmetry under anisotropic Weyl transformations can develop an anomaly in this symmetry at the quantum level. Under the transformations

$$
\delta_{\omega} N=z N \delta \omega, \quad \delta_{\omega} N_{i}=2 N_{i} \delta \omega, \quad \delta_{\omega} \gamma_{i j}=2 \gamma_{i j} \delta \omega,
$$

the anomaly will show up as a nonvanishing variation of the partition function $\mathcal{Z}\left[N, N_{i}, \gamma_{i j}\right]$, of the general form

$$
\delta_{\omega} \log \mathcal{Z}\left[N, N_{i}, \gamma_{i j}\right]=-\int d t d^{D} \mathbf{x} \sqrt{\gamma} N \mathfrak{A} \delta \omega
$$


where $\mathfrak{A}$ is now a function of $N, N_{i}$, and $\gamma_{i j}$.

We wish to determine what terms can arise in $\mathfrak{A}$. As in the relativistic case, this question is cohomological in nature. ${ }^{12}$ We introduce a nilpotent BRST operator $Q$, which acts on the metric multiplet via the infinitesimal anisotropic Weyl transformations (C.1), with $\delta \omega$ replaced by a Grassmann parameter $c$ of ghost number one. We can represent this operator as

$$
Q=c\left(z N \frac{\delta}{\delta N}+2 N_{i} \frac{\delta}{\delta N_{i}}+2 \gamma_{i j} \frac{\delta}{\delta \gamma_{i j}}\right) .
$$

Since $Q$ is nilpotent, the variation of the anomaly vanishes:

$$
Q \int d t d^{D} \mathbf{x} \sqrt{\gamma} N \mathfrak{A} c=-Q^{2} \log \mathcal{Z}=0
$$

This puts a constraint on the terms that can arise as $\mathfrak{A}$.

As usual, some of these terms can be removed by including appropriate counterterms. If a term in the anomaly can be expressed as the variation some local counterterm, this (gravitational) counterterm can be subtracted from the action, thereby eliminating the associated anomaly. Therefore the physical anomaly can be considered to lie in the cohomology of $Q$, at ghost number one. The number of possible independent terms (i.e., generalized central charges) in the anomaly will be determined by the dimension of this cohomology.

In the case of $2+1$ dimensions with $z=2$, the anomaly must be - on dimensional grounds - a sum of terms of dimension four, lying in the cohomology of $Q$. The list of possible terms is rather large; however, all but two are cohomologically trivial and can therefore be eliminated using local counterterms. The only ones that cannot be removed are:

$$
\hat{K}_{i j} \hat{K}^{i j}-\frac{1}{2} \hat{K}^{2}, \quad\left\{\hat{R}-\left(\frac{\nabla N}{N}\right)^{2}+\frac{\nabla^{2} N}{N}\right\}^{2} .
$$

We have seen in Section 4.2 that the first cohomology class in (C.5), quadratic in the extrinsic curvature $\hat{K}_{i j}$, indeed arises in the holographic computation of the anisotropic Weyl anomaly, but the second one does not. However, this term $\sim \hat{R}^{2}+\ldots$ is also non-trivial in the cohomology of $Q$, because $\sqrt{\gamma} N c R^{2}+\ldots$ cannot be obtained as the variation of another term (essentially because variations of all available terms give rise to derivatives). Hence, both classes should be expected to appear in the anomaly of generic $z=2$ field theories in $2+1$ dimensions.

In addition, we list $\mathfrak{A}$ for the five independent cocycles that contain only spatial derivatives, but are cohomologically trivial and can be eliminated by local counterterms:

$$
\frac{1}{N} \nabla^{2}\left[N\left(\hat{R}+\frac{\nabla^{2} N}{N}-\left(\frac{\nabla N}{N}\right)^{2}\right)\right],
$$

\footnotetext{
${ }^{12}$ The cohomological approach to the relativistic Weyl anomaly was developed in [67-69]; see [70], Chapter 22 , for a general review of this approach.
} 


$$
\begin{gathered}
\frac{1}{N} \nabla_{i}\left(\left[\hat{R}+\frac{\nabla^{2} N}{N}-\left(\frac{\nabla N}{N}\right)^{2}\right] \nabla^{i} N\right), \\
\frac{1}{N} \nabla^{2}\left[\nabla^{2} N-\frac{(\nabla N)^{2}}{N}\right] \\
\frac{1}{N} \nabla_{i}\left(\nabla^{i} N\left[\frac{\nabla^{2} N}{N}-\left(\frac{\nabla N}{N}\right)^{2}\right]-\frac{1}{2} \nabla^{i} \frac{(\nabla N)^{2}}{N}\right), \\
\frac{1}{N} \nabla_{i}\left[\nabla^{i} N\left(\frac{\nabla N}{N}\right)^{2}\right]
\end{gathered}
$$

This classification can be easily extended to include terms with time derivatives as well.

As usual, this cohomology analysis only reveals the complete list of terms which may in principle occur in the anomaly. Whether or not such terms are generated in a particular theory is a dynamical question, which requires an additional calculation.

\section{References}

[1] P. Hořava, Membranes at Quantum Criticality, JHEP 03 (2009) 020, arXiv:0812.4287.

[2] P. Hořava, Quantum Gravity at a Lifshitz Point, Phys. Rev. D79 (2009) 084008, arXiv:0901.3775.

[3] P. Hořava, General Covariance in Gravity at a Lifshitz Point, Class. Quant. Grav. 28 (2011) 114012, arXiv:1101.1081.

[4] M. Visser, Status of Hořava gravity: A personal perspective, J. Phys. Conf. Ser. 314 (2011) 012002, arXiv: 1103.5587.

[5] S. Mukohyama, Hořava-Lifshitz Cosmology: A Review, Class. Quant. Grav. 27 (2010) 223101, arXiv: 1007.5199.

[6] J. Ambjørn, J. Jurkiewicz, and R. Loll, Spectral Dimension of the Universe, Phys. Rev. Lett. 95 (2005) 171301, hep-th/0505113.

[7] J. Ambjørn, J. Jurkiewicz, and R. Loll, Quantum Gravity as Sum over Spacetimes, Lect. Notes Phys. 807 (2010) 59-124, 0906.3947.

[8] D. Benedetti and J. Henson, Spectral Geometry as a Probe of Quantum Spacetime, Phys.Rev. D80 (2009) 124036, 0911.0401.

[9] C. Anderson, S. Carlip, J. H. Cooperman, P. Hořava, R. Kommu, and P. R. Zulkowski, Quantizing Hor̆ava-Lifshitz Gravity via Causal Dynamical Triangulations, arXiv:1111.6634.

[10] S. A. Hartnoll, Lectures on Holographic Methods for Condensed Matter Physics, Class. Quant. Grav. 26 (2009) 224002, arXiv:0903.3246.

[11] J. McGreevy, Holographic Duality with a View toward Many-Body Physics, Adv. High Energy Phys. 2010 (2010) 723105, arXiv:0909.0518.

[12] S. Sachdev, What Can Gauge-Gravity Duality Teach us about Condensed Matter Physics?, arXiv: 1108.1197 . 
[13] V. E. Hubeny and M. Rangamani, A Holographic View on Physics out of Equilibrium, Adv.High Energy Phys. 2010 (2010) 297916, arXiv:1006.3675].

[14] D. T. Son, Toward an AdS/Cold Atoms Correspondence: A Geometric Realization of the Schrödinger Symmetry, Phys. Rev. D78 (2008) 046003, arXiv:0804.3972.

[15] K. Balasubramanian and J. McGreevy, Gravity Duals for Non-Relativistic CFTs, Phys. Rev. Lett. 101 (2008) 061601, arXiv:0804.4053.

[16] S. Kachru, X. Liu, and M. Mulligan, Gravity Duals of Lifshitz-like Fixed Points, Phys.Rev. D78 (2008) 106005, arXiv:0808.1725.

[17] R. Penrose and W. Rindler, Spinors and Space-Time, Vol. 2. Cambridge Univ. Press, 1986.

[18] P. Hořava and C. M. Melby-Thompson, Anisotropic Conformal Infinity, Gen. Rel. Grav. 43 (2010) 1391, arXiv:0909.3841.

[19] M. Henningson and K. Skenderis, The Holographic Weyl anomaly, JHEP 9807 (1998) 023, arXiv:hep-th/9806087.

[20] M. Duff, Twenty years of the Weyl anomaly, Class. Quant. Grav. 11 (1994) 1387-1404, arXiv:hep-th/9308075.

[21] V. Balasubramanian, E. G. Gimon, D. Minic, and J. Rahmfeld, Four-dimensional conformal supergravity from AdS space, Phys. Rev. D63 (2001) 104009, arXiv:hep-th/0007211.

[22] E. Fradkin and A. A. Tseytlin, Conformal Supergravity, Phys. Rept. 119 (1985) 233-362.

[23] I. Adam, I. V. Melnikov, and S. Theisen, A Non-Relativistic Weyl Anomaly, JHEP 0909 (2009) 130, arXiv:0907.2156.

[24] I. Bakas and D. Lüst, Axial Anomalies of Lifshitz Fermions, arXiv:1103.5693.

[25] I. Bakas, More on Axial Anomalies of Lifshitz Fermions, arXiv:1110.1332.

[26] K. Skenderis, Lecture notes on holographic renormalization, Class. Quant. Grav. 19 (2002) 5849, arXiv:hep-th/0209067.

[27] J. Maldacena, Einstein Gravity from Conformal Gravity, arXiv:1105.5632.

[28] J. M. Maldacena, Non-Gaussian features of primordial fluctuations in single field inflationary models, JHEP 0305 (2003) 013, astro-ph/0210603.

[29] D. Harlow and D. Stanford, Operator Dictionaries and Wave Functions in AdS/CFT and $d S / C F T$, arXiv:1104.2621.

[30] P. McFadden and K. Skenderis, Holography for Cosmology, Phys.Rev. D81 (2010) 021301, arXiv:0907.5542.

[31] P. McFadden and K. Skenderis, Cosmological 3-point Correlators from Holography, JCAP 1106 (2011) 030, arXiv:1104.3894.

[32] A. Bzowski, P. McFadden, and K. Skenderis, Holographic Predictions for Cosmological 3-point Functions, JHEP 1203 (2012) 091, arXiv:1112.1967.

[33] P. Hořava and C. M. Melby-Thompson, General Covariance in Quantum Gravity at a Lifshitz Point, Phys. Rev. D82 (2010) 064027, arXiv:1007.2410. 
[34] D. Blas, O. Pujolàs, and S. Sibiryakov, Consistent Extension of Hořava Gravity, Phys. Rev. Lett. 104 (2010) 181302, arXiv:0909.3525.

[35] D. Blas, O. Pujolàs, and S. Sibiryakov, Models of non-relativistic quantum gravity: The Good, the bad and the healthy, JHEP 1104 (2011) 018, arXiv:1007.3503.

[36] M. Taylor, Non-relativistic Holography, arXiv:0812.0530.

[37] K. Balasubramanian and K. Narayan, Lifshitz Spacetimes from AdS Null and Cosmological Solutions, JHEP 1008 (2010) 014, arXiv:1005.3291.

[38] A. Donos and J. P. Gauntlett, Lifshitz Solutions of D=10 and D=11 Supergravity, JHEP 1012 (2010) 002, arXiv:1008.2062.

[39] R. Gregory, S. L. Parameswaran, G. Tasinato, and I. Zavala, Lifshitz Solutions in Supergravity and String Theory, JHEP 1012 (2010) 047, arXiv:1009.3445.

[40] A. Donos, J. P. Gauntlett, N. Kim, and O. Varela, Wrapped M5-Branes, Consistent Truncations and AdS/CMT, JHEP 1012 (2010) 003, arXiv:1009.3805.

[41] S. W. Hawking and G. F. R. Ellis, The Large Scale Structure of Space-time. Cambridge Univ. Press, 1973.

[42] S. F. Ross, Holography for asymptotically locally Lifshitz spacetimes, Class. Quant. Grav. 28 (2011) 215019, arXiv:1107.4451.

[43] J. M. Maldacena, TASI 2003 Lectures on AdS/CFT, arXiv:hep-th/0309246.

[44] J. Polchinski, Introduction to Gauge/Gravity Duality, arXiv:1010.6134.

[45] V. Balasubramanian and P. Kraus, A Stress Tensor for Anti-de Sitter Gravity, Commun. Math. Phys. 208 (1999) 413-428, arXiv:hep-th/9902121.

[46] P. Kraus, F. Larsen, and R. Siebelink, The Gravitational Action in Asymptotically AdS and Flat Spacetimes, Nucl. Phys. B563 (1999) 259-278, arXiv:hep-th/9906127.

[47] S. de Haro, S. N. Solodukhin, and K. Skenderis, Holographic Reconstruction of Spacetime and Renormalization in the AdS/CFT Correspondence, Commun. Math. Phys. 217 (2001) 595-622, arXiv:hep-th/0002230.

[48] M. Bianchi, D. Z. Freedman, and K. Skenderis, Holographic Renormalization, Nucl.Phys. B631 (2002) 159-194, arXiv:hep-th/0112119.

[49] J. de Boer, E. P. Verlinde, and H. L. Verlinde, On the Holographic Renormalization Group, JHEP 0008 (2000) 003, arXiv:hep-th/9912012.

[50] J. de Boer, The Holographic renormalization group, Fortsch.Phys. 49 (2001) 339-358, arXiv:hep-th/0101026.

[51] M. Fukuma, S. Matsuura, and T. Sakai, Holographic renormalization group, Prog. Theor. Phys. 109 (2003) 489, arXiv:hep-th/0212314.

[52] R. Mann and R. McNees, Holographic Renormalization for Asymptotically Lifshitz Spacetimes, JHEP 1110 (2011) 129, arXiv:1107.5792.

[53] M. Baggio, J. de Boer, and K. Holsheimer, Hamilton-Jacobi Renormalization for Lifshitz Spacetime, arXiv:1107.5562. 
[54] I. Papadimitriou and K. Skenderis, AdS / CFT correspondence and geometry, arXiv:hep-th/0404176.

[55] I. Papadimitriou and K. Skenderis, Correlation functions in holographic RG flows, JHEP 0410 (2004) 075, arXiv:hep-th/0407071.

[56] C. Fefferman and C. R. Graham, Conformal Invariants, in: Elie Cartan et les Mathématiques d'aujourd'hui, Astérisque (1985) 95.

[57] C. R. Graham, Volume and Area Renormalizations for Conformally Compact Einstein Metrics, arXiv:math/9909042.

[58] C. R. Graham and E. Witten, Conformal Anomaly of Submanifold Observables in AdS/CFT Correspondence, Nucl. Phys. B546 (1999) 52-64, arXiv:hep-th/9901021.

[59] D. Vernieri and T. P. Sotiriou, Hořava-Lifshitz Gravity: Detailed Balance Revisited, arXiv:1112.3385.

[60] P. Hořava, Gravity at a Lifshitz Point, review talk at Strings 2009 (Roma, Italy) June 25, 2009 (unpublished), http://strings2009.roma2.infn.it/talks/Horava_Strings09.pdf].

[61] K. T. Grosvenor, P. Hořava, and C. M. Melby-Thompson, Quantum Gravity with Anisotropic Scaling Near the Schwarzschild Horizon, to appear.

[62] C. Xu and P. Hořava, Emergent Gravity at a Lifshitz Point from a Bose Liquid on the Lattice, Phys. Rev. D81 (2010) 104033, arXiv:1003.0009.

[63] G. Volovik, Topology of Quantum Vacuum, arXiv:1111.4627.

[64] S. F. Ross and O. Saremi, Holographic stress tensor for non-relativistic theories, JHEP 0909 (2009) 009, arXiv:0907.1846.

[65] H. Braviner, R. Gregory, and S. F. Ross, Flows Involving Lifshitz Solutions, Class. Quant. Grav. 28 (2011) 225028, arXiv:1108.3067.

[66] M. C. Cheng, S. A. Hartnoll, and C. A. Keeler, Deformations of Lifshitz holography, JHEP 1003 (2010) 062, arXiv:0912.2784.

[67] L. Bonora, P. Pasti, and M. Bregola, Weyl Cocycles, Class. Quant. Grav. 3 (1986) 635.

[68] W. A. Bardeen and B. Zumino, Consistent and Covariant Anomalies in Gauge and Gravitational Theories, Nucl. Phys. B244 (1984) 421.

[69] S. Deser and A. Schwimmer, Geometric classification of conformal anomalies in arbitrary dimensions, Phys. Lett. B309 (1993) 279, arXiv:hep-th/9302047.

[70] S. Weinberg, The Quantum Theory of Fields, Vol. 2. Cambridge Univ. Press, 1996. 